TRANSACTIONS OF THE

AMERICAN MATHEMATICAL SOCIETY

Volume 358, Number 10, October 2006, Pages 4653-4687

S $0002-9947(06) 03894-3$

Article electronically published on May 17, 2006

\title{
ON THE NON-UNITARY UNRAMIFIED DUAL FOR CLASSICAL $p$-ADIC GROUPS
}

\author{
GORAN MUIĆ
}

\begin{abstract}
In this paper we give a Zelevinsky type classification of unramified irreducible representations of split classical groups.
\end{abstract}

\section{INTRODUCTION}

Generic irreducible representations of quasi-split classical groups were classified by the author in (M1, M2, M3]) for local non-archimedean fields of characteristic zero. The classification consists of three steps. The first one is the deepest. It gives the characterization of generic discrete series in terms of poles of local $\mathrm{L}^{-}$ functions (see M2, Theorem 3.1). The $L$-functions used are defined by Shahidi in (Sh1], Sh2]) assuming that the field has characteristic zero. The second step is to realize tempered irreducible representation as a subrepresentation of a representation induced from generic discrete series. This step follows from the general theory of tempered representations. The final step is to prove that Langlands quotient is generic if and only if its standard module is irreducible. In (M1, M2] we give a fairly general procedure that reduces the last step to the first step. Although this reduction does not depend on the characteristic of the field, the actual determination of the reducibility of standard modules in ([M1, [M2, [M3]) is done in terms of $L$-functions and thus it depends on the assumption that the field has characteristic zero. A similar approach is adopted in MSh to obtain similar results for a general split reductive group and its generic Iwahori-fixed representations again for fields of characteristic zero. In [MSh, we use a result from [BM1 about a well-known duality (under Iwahori-Matsumoto involution) between generic and unramified representations to obtain results about unramified representations from those for generic representations, but only for the fields of characteristic zero. This motivates our present approach.

The purpose of this paper is twofold. First, we prove that this type of classification exists over any non-archimedean local field $F$ of characteristic different than 2, for unramified representations of split classical groups $S p(n, F)$ (symplectic group of rank $n), S O(2 n+1, F)$ (split special odd-orthogonal group of rank $n$ ), and $O(2 n, F)$ (split special even-orthogonal group of rank $n$ ). Thus, we remove the assumption that the field $F$ is of characteristic zero. Second, for the theory of automorphic forms, unramified representations are fundamental objects to study and one wants to know more about them, especially about embeddings into principal series and degenerate principal series and structure of Jacquet modules. The

Received by the editors April 6, 2004 and, in revised form, November 22, 2004.

2000 Mathematics Subject Classification. Primary 22E35, 22E50; Secondary 11F70.

(C) 2006 American Mathematical Society Reverts to public domain 28 years from publication 
present paper addresses both problems in a simple and consistent way relying only on the following: Bernstein-Zelevinsky theory ([BZ1, [BZ2], ZZ] $)$, the Geometric lemma of [BZ1] written for our groups in [T2, [B], and a result of Waldspurger ( $\mathrm{MVW}]$ ) that describes the conragredient of a representation. Having written the paper this way, one could also expect to extend the results of the present paper to the case of metaplectic groups as soon as one extends the geometric lemma there.

Now, describe our results and the content of the paper in more detail. We write $\nu$ for the normalized absolute value of $F$. Let $\chi$ be a character of $F^{\times}$. Then we can decompose it uniquely as follows: $\chi=\nu^{e(\chi)} \chi^{u}, \chi^{u}$ is a unitary character and $e(\chi) \in \mathbb{R}$. We write $G_{n}=S p(n, F), S O(2 n+1, F)$, or $O(2 n, F)$. Let $\sigma \in \operatorname{Irr} G_{n}$ be an unramified representation. Then we write write $\operatorname{Jacq}_{(1, \ldots, 1 ; 0)}(\sigma)$ for its Jacquet module with respect to the Borel parabolic subgroup. We say that $\sigma$ is negative if for any irreducible subquotient $\chi_{1} \otimes \chi_{2} \otimes \cdots \otimes \chi_{n}$ of $\operatorname{Jacq}_{(1, \ldots, 1 ; 0)}(\sigma)$ we have

$$
\left\{\begin{array}{l}
e\left(\chi_{1}\right) \leq 0, \\
e\left(\chi_{1}\right)+e\left(\chi_{2}\right) \leq 0, \\
\cdots \\
e\left(\chi_{1}\right)+e\left(\chi_{2}\right)+\cdots+e\left(\chi_{n}\right) \leq 0 .
\end{array}\right.
$$

We say that $\sigma$ is strictly negative if all inequalities are strict. Negative and strictly negative representations play a role of tempered and discrete series representations, respectively. In fact, for connected groups the Iwahori-Matsumoto involution of strictly negative (resp. negative) representation is a discrete series (resp. tempered representation) (the fact that we do not use in the present paper). The first classification result is the analogue of the main theorem of $\mathrm{M} 2$ proved for the characteristic zero field $F$ using $L$-functions of Shahidi (the last step in the classification of generic representations as explained above). It can be formulated as follows (see Theorem 4.3).

Theorem. Let $\sigma \in \operatorname{Irr} G_{n}$ be an unramified representation. Then there exists a unique up to permutation sequence of unramified characters $\chi_{1} \mathbf{1}_{G L\left(n_{1}, F\right)}, \ldots$, $\chi_{k} \mathbf{1}_{G L\left(n_{k}, F\right)}, e\left(\chi_{i}\right)>0(i=1, \ldots, k)$, and a unique negative representation $\sigma_{\text {neg }}$ such that $\sigma$ is fully induced from $\chi_{1} \mathbf{1}_{G L\left(n_{1}, F\right)} \otimes \cdots \otimes \chi_{k} \mathbf{1}_{G L\left(n_{k}, F\right)} \otimes \sigma_{n e g}$ :

$$
\sigma \simeq \chi_{1} \mathbf{1}_{G L\left(n_{1}, F\right)} \times \cdots \times \chi_{k} \mathbf{1}_{G L\left(n_{k}, F\right)} \rtimes \sigma_{\text {neg }} .
$$

Negative representations are classified in terms of strictly negative as follows.

Theorem. Let $\sigma \in \operatorname{Irr} G_{n}$ be a negative unramified representation. Then there exists a sequence of unitary unramified characters $\chi_{1} \mathbf{1}_{G L\left(n_{1}, F\right)}, \ldots, \chi_{k} \mathbf{1}_{G L\left(n_{k}, F\right)}$ (unique up to permutation and taking inverses) and a unique strictly negative representation $\sigma_{s n}$ such that $\sigma$ is embedded into a representation induced from $\chi_{1} \mathbf{1}_{G L\left(n_{1}, F\right)} \otimes \cdots \otimes \chi_{k} \mathbf{1}_{G L\left(n_{k}, F\right)} \otimes \sigma_{n e g}:$

$$
\sigma \hookrightarrow \chi_{1} \mathbf{1}_{G L\left(n_{1}, F\right)} \times \cdots \times \chi_{k} \mathbf{1}_{G L\left(n_{k}, F\right)} \rtimes \sigma_{s n} .
$$

This is an analogue of step two in the classification of generic representations, but it is in fact much more deeper. Let us explain in more detail. There are two ways to obtain this theorem. One way could be the following. We apply the usual theory of tempered representations (where except Jacquet modules we use the fact that since parabolic induction carries unitary representations into unitary ones) and then use a deep result of Barbasch-Moy (BM2], BM3]) on preserving unitarity under the 
Iwahori-Matsumoto involution. In this way one could obtain this theorem using the full power of geometric considerations of Kazhdan-Lusztig KL. Instead we use a different and more elementary approach based only on Jacquet modules (Theorem 4.4 and Theorem 6.1). In fact the existence of embedding is fairly easy to prove (see Theorem 4.4), but the uniqueness is established using the full classification of strongly negative representations completed in Section 5 (see Theorem 6.1).

Finally, we discuss the analogue of step one for generic representations. This part occupies most of the paper (see Section 5). Thus, we describe the set of equivalence classes of strongly negative irreducible unramified representations of $G_{n}$, denoted by $\operatorname{Irr}_{s n}\left(G_{n}\right)$.

First, we define the notion of the Jordan block following Moeglin (cf. Moe]) as the pair $(m, \chi)$, where $m \in \mathbb{Z}_{>0}$ and $\chi \in\left\{\mathbf{1}, \chi_{0}\right\}$ ( $\chi_{0}$ is the unique non-trivial unramified quadratic character of $F^{\times}$), such that $m$ is even if and only if we deal with odd-orthogonal groups (and odd if and only if we deal with symplectic or even-orthogonal groups). We write $\operatorname{Jord}(n)$ for the collection of all sets of Jordan blocks Jord $:=\{(m, \chi)\}$ such that

$$
\sum_{(m, \chi) \in \mathrm{Jord}} m=\left\{\begin{array}{l}
2 n \quad \text { if } G_{n}=S O(2 n+1, F), O(2 n, F) \\
2 n+1 \quad \text { if } G_{n}=S p(n, F) .
\end{array}\right.
$$

Let $\operatorname{Jord}(\chi)$ be the set of all $m \in \mathbb{Z}_{>0} \operatorname{such}$ that $(m, \chi) \in$ Jord. We also require that \# $\operatorname{Jord}(\chi)$ (resp., \# $\left.\operatorname{Jord}\left(\chi_{0}\right)\right)$ is even for $G_{n}=O(2 n, F)\left(\operatorname{resp} ., G_{n}=S p(n, F)\right)$. Then we write $\operatorname{Jord}(\chi)$ according to the character $\chi$ (the case $l=0$ or $k=0$ is not excluded):

$$
\left\{\begin{array}{l}
\chi=1: \quad 2 m_{1}+1<2 m_{2}+1<\cdots<2 m_{l}+1 \\
\chi=\chi_{0}: \quad 2 n_{1}+1<2 n_{2}+1<\cdots<2 n_{k}+1
\end{array}\right.
$$

where $m_{i}, n_{j} \in \mathbb{Z}_{\geq 0}\left(G_{n}=S p(n, F), O(2 n, F)\right)$ or $m_{i}, n_{j} \in \frac{1}{2}+\mathbb{Z}_{\geq 0}\left(G_{n}=\right.$ $S O(2 n+1, F))$.

Next, we associate an unramified representation $\sigma$ (Jord) defined as a unique irreducible unramified subquotient of

$$
\begin{array}{r}
*) \quad \nu^{\left(m_{l-1}-m_{l}\right) / 2} \mathbf{1}_{G L\left(m_{l-1}+m_{l}+1, F\right)} \times \nu^{\left(m_{l-3}-m_{l-2}\right) / 2} \mathbf{1}_{G L\left(m_{l-3}+m_{l-2}+1, F\right)} \\
\times \cdots \times \nu^{\left(n_{k-1}-n_{k}\right) / 2} \chi_{0} \mathbf{1}_{G L\left(n_{k-1}+n_{k}+1, F\right)} \times \nu^{\left(n_{k-3}-n_{k-2}\right) / 2} \chi_{0} \mathbf{1}_{G L\left(n_{k-3}+n_{k-2}+1, F\right)} \\
\times \cdots \\
\times \sigma_{0}(\mathrm{Jord}),
\end{array}
$$

where $\sigma_{0}$ (Jord) is a unique unramified irreducible subquotient of the following induced representation:

(i) $\nu^{\left(1-m_{1}\right) / 2} \mathbf{1}_{G L(m, F)} \rtimes \mathbf{1}, l \in 2 \mathbb{Z}+1, G_{n}=S p(n, F)$;

(ii) $\nu^{\left(1 / 2-m_{1}\right) / 2} \mathbf{1}_{G L\left(m_{1}+1 / 2, F\right)} \times \nu^{\left(1 / 2-n_{1}\right) / 2} \chi_{0} \mathbf{1}_{G L\left(n_{1}+1 / 2, F\right)} \rtimes \mathbf{1}, k, l \in 2 \mathbb{Z}+1$, $G_{n}=S O(2 n+1, F)$

(iii) $\nu^{\left(1 / 2-m_{1}\right) / 2} \mathbf{1}_{G L\left(m_{1}+1 / 2, F\right)} \rtimes \mathbf{1}, k \in 2 \mathbb{Z}+1, l \in 2 \mathbb{Z}, G_{n}=S O(2 n+1, F)$;

(iv) $\nu^{\left(1 / 2-n_{1}\right) / 2} \chi_{0} \mathbf{1}_{G L\left(n_{1}+1 / 2, F\right)} \rtimes \mathbf{1}, k \in 2 \mathbb{Z}, l \in 2 \mathbb{Z}+1, G_{n}=S O(2 n+1, F)$;

(v) $\mathbf{1} \in \operatorname{Irr} G_{0}, k, l \in 2 \mathbb{Z}$.

In fact, $\sigma$ (Jord) and $\sigma_{0}$ (Jord) are both strongly negative and irreducible subrepresentations of their defining induced representations given above. This is proved in Lemmas 5.1-5.5. We should note that the proof of strong negativity of $\sigma$ (Jord) is a lot simpler and different than the proofs of the corresponding facts in ([T3], [MT] 
needed in the construction of discrete series. Finally, in Lemma 5.7 we complete the proof of the following theorem (Theorem 5.1).

Theorem. Let $n \in \mathbb{Z}_{>0}$. The correspondence $\operatorname{Jord}(n) \rightarrow \operatorname{Irr}_{s n}\left(G_{n}\right)$ given by Jord $\rightsquigarrow \sigma$ (Jord) is a well-defined bijection.

This completes our classification of unramified representations.

I would like to thank M. Tadić for encouraging me to write the present paper and for his help with Theorem 3.2. Many techniques used in this paper I learned and adapted from his papers. I would like to thank S. Rallis for turning my attention to the problem of the determination of Jacquet modules of unramified representations during my visit to the Ohio State University during November of 1998. I would also like to thank the referee, who helped to improve the style of the presentation.

\section{Preliminaries}

Let $F$ be a nonarchimedean field of characteristic different from 2. We write $\mathcal{O}$ for the maximal compact subring of $F$ and $q$ for the number of elements in the corresponding residue field of $\mathcal{O}$. We write $\nu$ for the normalized absolute value of $F$. Let $\chi$ be a character of $F^{\times}$. Then we can uniquely write $\chi=\nu^{e(\chi)} \chi^{u}$, where $\chi^{u}$ is a unitary character and $e(\chi) \in \mathbb{R}$.

Let $\mathbb{Z}, \mathbb{R}$, and $\mathbb{C}$ be the ring of rational integers, the field of real numbers, and the field of complex numbers, respectively.

Assume that $G$ is a unimodular $l$-group and $K, M, N, P$ its closed subgroups such that $K$ is compact open, $P=M \rtimes N, P \cap K=(M \cap K)(N \cap K)$, and $G=K P$ (cf. BZ2).

Finally we assume that $N$ is a union of its open-compact subgroups. We write $\operatorname{Ind}_{P}^{G}$ and $\operatorname{Jacq}_{P}^{G}$ for the normalized parabolic induction and normalized Jacquet module. They are related by the Frobenius reciprocity:

$$
\operatorname{Hom}_{G}\left(\sigma, \operatorname{Ind}_{P}^{G}\left(\sigma^{\prime} \otimes \mathbf{1}_{N}\right)\right) \simeq \operatorname{Hom}_{M}\left(\operatorname{Jacq}_{P}^{G}(\sigma), \sigma^{\prime}\right) .
$$

Assume that $\left(\sigma, V_{\sigma}\right)$ is a smooth representation of $G$. We say that $\sigma$ is $K_{-}$ spherical if $\left(V_{\sigma}\right)^{K} \neq 0$. Let the pair $(G, K)$ be given by $(n>0)$ :

- $G_{n}=S p(n, F)$ (symplectic group of rank $\left.n\right) ; K=S p(n, \mathcal{O})$.

- $G_{n}=S O(2 n+1, F)$ (split special odd-orthogonal group of rank $n$ ); $K=$ $S O(2 n+1, \mathcal{O})$.

- $G_{n}=O(2 n, F)$ (split special even-orthogonal group of rank $n$ ); $K=$ $O(2 n, \mathcal{O})$.

- $(G L(n, F), G L(n, \mathcal{O}))$.

Then we say that $K$-spherical representation of $G$ is unramified. The pair $(G, K)$ satisfies all of the above assumptions (cf. $\mathrm{Car}$ ). Finally, we let $G_{0}$ be the trivial group. We also call its unique irreducible representation $\mathbf{1}$ unramified.

Going back to the general pair $(G, K)$ satisfying the above conditions we prove the next simple lemma that we will use several times.

Lemma 1.1. (i) Assume $\sigma^{\prime}$ is an $M \cap K$-spherical smooth representation of $M$. Then $\operatorname{Ind}_{M N}^{G}\left(\sigma^{\prime} \otimes \mathbf{1}_{N}\right)$ is $K$-spherical.

(ii) Assume that $\sigma$ is a $K$-spherical smooth representation of $G$, and $\sigma$ is a subquotient of $\operatorname{Ind}_{M N}^{G}\left(\sigma^{\prime} \otimes \mathbf{1}_{N}\right)$, for some smooth representation $\sigma^{\prime}$ of $M$. Then $\sigma^{\prime}$ is $M \cap K$-spherical. 
Proof. We prove (ii). (i) has a similar but simpler proof. Since $K$ is compact and open, the functor $W \rightsquigarrow W^{K}$ from the category of smooth $G$-representations into complex vector spaces is exact [BZ2]. Therefore, the assumption implies that there is a function $f \neq 0$ in the space of $\operatorname{Ind}_{M N}^{G}\left(\sigma^{\prime} \otimes \mathbf{1}_{N}\right)$ such that $f(g k)=f(g)$, for all $g \in G, k \in K$. More precisely, we have $f: G \rightarrow V_{\sigma^{\prime}}$ and

$$
f(m n k)=\delta_{P}(m)^{1 / 2} \sigma^{\prime}(m) v, m \in M, n \in N, k \in K,
$$

where $v:=f(1) \in V_{\sigma^{\prime}}$. Since $G=P K$ we see that $v \neq 0$. Finally, since $P \cap K=$ $(M \cap K)(N \cap K)$, we see that $v \in\left(V_{\sigma^{\prime}}\right)^{M \cap K}$.

\section{UnRAmified REPRESENTATIONS OF $G L(n, F)$}

In this section we recall some results from [Ze]. First, if $\chi$ is a character of $F^{\times}$, then we can consider it as a character of $G L(n, F)$ as $g \rightsquigarrow \chi(\operatorname{det} g)$. We denote this character as $\chi \mathbf{1}_{n}$. In this way we get $1-1$ correspondence between characters of $G L(n, F)$ and $F^{\times}$that preserves the sets of unitary and unramified characters, respectively. Further, we have the following lemma (cf. [Ze]).

Lemma 2.1. Assume that $\chi, \chi^{\prime}$ are characters of $F^{\times}$and $\alpha, \beta, \alpha^{\prime}, \beta^{\prime} \in \mathbb{R}$ such that $\alpha+\beta \in \mathbb{Z}_{\geq 0}$ and $\alpha^{\prime}+\beta^{\prime} \in \mathbb{Z}_{\geq 0}$ Then we have the following:

$$
\left\{\begin{array}{l}
\chi \nu^{(\alpha-\beta) / 2} \mathbf{1}_{\alpha+\beta+1} \hookrightarrow \nu^{-\beta} \chi \times \nu^{-\beta+1} \chi \times \cdots \times \nu^{\alpha} \chi, \\
\nu^{\alpha} \chi \times \nu^{\alpha-1} \chi \times \cdots \times \nu^{-\beta} \chi \rightarrow \chi \nu^{(\alpha-\beta) / 2} \mathbf{1}_{\alpha+\beta+1} .
\end{array}\right.
$$

We write $\zeta(-\beta, \alpha, \chi):=\chi \nu^{(\alpha-\beta) / 2} \mathbf{1}_{\alpha+\beta+1}$. Moreover, $\zeta(-\beta, \alpha, \chi) \times \zeta\left(-\beta^{\prime}, \alpha^{\prime}, \chi^{\prime}\right)$ is reducible if and only if $\chi=\chi^{\prime}, \alpha-\alpha^{\prime} \in \mathbb{Z}$, and one of the following holds:

$$
\left\{\begin{array}{l}
-\beta \leq-\beta^{\prime}-1 \leq \alpha<\alpha^{\prime} \\
-\beta^{\prime} \leq-\beta-1 \leq \alpha^{\prime}<\alpha
\end{array}\right.
$$

Moreover, if $\zeta(-\beta, \alpha, \chi) \times \zeta\left(-\beta^{\prime}, \alpha^{\prime}, \chi^{\prime}\right)$ reduces, then it has length two and contains $\zeta\left(-\beta^{\prime}, \alpha, \chi\right) \times \zeta\left(-\beta, \alpha^{\prime}, \chi\right)$ in its composition series with multiplicity one. More precisely,

$$
\zeta\left(-\beta^{\prime}, \alpha, \chi\right) \times \zeta\left(-\beta, \alpha^{\prime}, \chi\right) \hookrightarrow \begin{cases}\zeta(-\beta, \alpha, \chi) \times \zeta\left(-\beta^{\prime}, \alpha^{\prime}, \chi\right), & \alpha<\alpha^{\prime}, \\ \zeta\left(-\beta^{\prime}, \alpha^{\prime}, \chi\right) \times \zeta(-\beta, \alpha, \chi), & \alpha^{\prime}<\alpha,\end{cases}
$$

and

$$
\zeta\left(-\beta^{\prime}, \alpha, \chi\right) \times \zeta\left(-\beta, \alpha^{\prime}, \chi\right) \nleftarrow \begin{cases}\zeta(-\beta, \alpha, \chi) \times \zeta\left(-\beta^{\prime}, \alpha^{\prime}, \chi\right), & \alpha^{\prime}<\alpha, \\ \zeta\left(-\beta^{\prime}, \alpha^{\prime}, \chi\right) \times \zeta(-\beta, \alpha, \chi), & \alpha<\alpha^{\prime} .\end{cases}
$$

(We omit $\zeta\left(-\beta^{\prime}, \alpha, \chi\right)$ if $\alpha=-\beta^{\prime}-1$, and $\zeta\left(-\beta^{\prime}, \alpha^{\prime}, \chi\right)$ if $\alpha^{\prime}=-\beta-1$. In this paper $\nleftarrow$ and $\rightarrow$ stand for epimorphisms in the direction of the arrows.)

Finally, we turn our attention to unramified representations. The next theorem represents the classification of unramified representations, and it can be easily seen from $[\mathrm{Ze}$.

Theorem 2.1. $\quad$ (i) Assume that $\left(\chi_{1} \mathbf{1}_{n_{1}}, \ldots, \chi_{k} \mathbf{1}_{n_{k}}\right)$ is a sequence of characters. Then $\chi_{1} \mathbf{1}_{G L\left(n_{1}\right)} \times \cdots \times \chi_{k} \mathbf{1}_{G L\left(n_{k}\right)}$ reduces if and only if there exists $i<j$ such that $\chi_{i} \mathbf{1}_{G L\left(n_{i}\right)} \times \chi_{j} \mathbf{1}_{G L\left(n_{j}\right)}$ reduces. We note that $\chi_{i} \mathbf{1}_{G L\left(n_{i}\right)}=$ $\zeta\left(-\left(n_{i}-1\right) / 2,\left(n_{i}-1\right) / 2, \chi\right)$. 
(ii) Assume that $\pi \in \operatorname{Irr} G L(n, F)$ is an unramified representation (that is, the space of $G L(n, \mathcal{O})$-invariants in the space of $\pi$ is non-zero). Then up to permutation there exist a unique sequence of unramified characters $\left(\chi_{1} \mathbf{1}_{n_{1}}, \ldots, \chi_{k} \mathbf{1}_{n_{k}}\right), n_{1}+\cdots+n_{k}=n$, such that $\sigma \simeq \chi_{1} \mathbf{1}_{G L\left(n_{1}\right)} \times \cdots \times$ $\chi_{k} \mathbf{1}_{G L\left(n_{k}\right)}$.

In Section 4 we prove the analogue of Theorem 2.1 for classical groups.

\section{Results of TAdić AND REDUCIBILITy OF THE RANK-ONE GENERALIZED PRINCIPAL SERIES}

In this section we compute the Jacquet module following Tadić (cf. [T2, B]). Let $R\left(G_{n}\right)$ be the Grothendieck group of admissible representations of finite length of $G_{n}$. Put

$$
R(G)=\bigoplus_{n \geq 0} R\left(G_{n}\right)
$$

We write $\geq$ or $\leq$ for the natural order on $R(G)$. In more detail, $\pi_{1} \leq \pi_{2}, \pi_{1}, \pi_{2} \in$ $R(G)$, if and only if $\pi_{2}-\pi_{1}$ is a linear combination of irreducible representations with non-negative coefficients. If $\pi \in R(G)$ and $\pi^{\prime} \in \operatorname{Irr} G$, we write $\operatorname{Mult}\left(\pi^{\prime}, \pi\right)$ for the largest integer $m$ such that

$$
\left\{\begin{array}{l}
m \cdot \pi^{\prime} \leq \pi, \\
(m+1) \cdot \pi^{\prime} \not \leq \pi
\end{array}\right.
$$

Clearly, $\operatorname{Mult}\left(\pi^{\prime}, \pi\right) \geq 0$. We also define

$$
R(G L)=\bigoplus_{n \geq 0} R(G L(n, F))
$$

Let $\sigma \in \operatorname{Irr} G_{n}$. Then for each standard proper maximal parabolic subgroup (cf. MVW] $P_{j}$ with Levi factor $G L(j, F) \times G_{n-j}, 1 \leq j \leq n$, we can identify (normalized Jacquet module) $\operatorname{Jacq}_{(j ; n-j)}(\sigma):=\operatorname{Jacq}_{P_{j}}^{G_{n}}(\sigma)$ with its semisimplification in $R(G L(j, F)) \otimes R\left(G_{n-j}\right)$. Thus, we consider

$$
\mu^{*}(\sigma)=1 \otimes \sigma+\sum_{j=1}^{n} \operatorname{Jacq}_{(j ; n-j)}(\sigma) \in R(G L) \otimes R(G) .
$$

Now, the basic result of Tadić (cf. T2, B]) is the following theorem.

Theorem 3.1. Let $\sigma \in \operatorname{Irr} G_{n}$. We decompose $R(G)$ into irreducible constituents (with repetitions possible):

$$
\mu^{*}(\sigma)=\sum_{\zeta, \sigma^{\prime}} \zeta \otimes \sigma^{\prime}
$$

Assume that $\alpha, \beta \in \mathbb{R}, \alpha+\beta+1 \in \mathbb{Z}_{>0}$, and $\chi$ is a character of $F^{\times}$. Then we have

$$
\begin{aligned}
& \mu^{*}(\zeta(-\beta, \alpha, \chi) \rtimes \sigma) \\
& =\sum_{\zeta, \sigma^{\prime}} \sum_{i=0}^{\alpha+\beta+1} \sum_{j=0}^{i} \zeta\left(-\alpha, \beta-i, \chi^{-1}\right) \times \zeta(-\beta, j-\beta-1, \chi) \times \zeta \\
& \otimes \zeta(j-\beta, i-\beta-1, \chi) \rtimes \sigma^{\prime} .
\end{aligned}
$$

(We omit $\zeta(a, b, \chi)$ if $a>b$.) 
We will also need the next theorem, which is essentially proved in [T1].

Theorem 3.2. Let $\mathbf{1} \in \operatorname{Irr} G_{0}$ be the trivial representation. Assume that $\alpha, \beta \in \mathbb{R}$, $\alpha+\beta \in \mathbb{Z}_{\geq 0}$, and $\chi$ is a unitary character of $F^{\times}$. Then $\zeta(-\beta, \alpha, \chi) \rtimes \mathbf{1}$ reduces if and only if $\nu^{i} \chi \rtimes \mathbf{1}$ reduces for some $i,-\alpha \leq i \leq \beta, \beta-i \in \mathbb{Z}$.

Proof. In [T1, Tadić computes the reducibility of generalized principal $\delta(-\beta, \alpha, \chi) \rtimes$ 1 series where $\delta(-\beta, \alpha, \chi)$ is an essentially square-integrable representation being a subquotient of the same principal series as $\zeta(-\beta, \alpha, \chi)$. The end result has the analogous formulation. It is easy to adapt his proof to see that it can be applied to our $\zeta(-\beta, \alpha, \chi) \rtimes \mathbf{1}$ since his proof just uses Theorem 3.1, Bernstein-Zelevinsky theory (cf. [BZ1], Ze]), very simple and general facts about the reducibility of induced representations (see Section 3 of [T1]), and the fact that the normalized induction carries unitarizable representations into unitarizable representations.

The next result is a well-known result on rank-one reducibilities.

Theorem 3.3. Let $\mathbf{1} \in \operatorname{Irr} G_{0}$ be the trivial representation. Put $I(s, \chi)=\nu^{s} \chi \rtimes \mathbf{1}$ $(s \in \mathbb{R}, \chi$ unitary and unramified). Then we have the following:

(i) $I(s, \chi)$ has the same composition series as $I\left(-s, \chi^{-1}\right)$.

(ii) If $\chi^{2} \neq \mathbf{1}$, then $I(s, \chi)$ is irreducible for all $s \in \mathbb{R}$.

(iii) Assume $\chi^{2}=\mathbf{1}$. (This implies that $\chi=\mathbf{1}$ or $\chi=\chi_{0}:=\nu^{\pi \sqrt{-1} / \ln (q)}$.) Then $I(s, \chi)$ is reducible if the one of the following holds:

- $s= \pm 1 / 2$, for $S O(3, F)$.

- $s=0$, for $O(2, F)$.

- $s=0, \chi=\chi_{0}$ or $s= \pm 1, \chi=\mathbf{1}$, for $S p(1, F)=S L(2, F)$.

(iv) If $I(s, \chi)(s>0)$ reduces, then its unique irreducible quotient is unramified. Hence also is a unique irreducible subrepresentation of $I(-s, \chi)$. (We remind the reader that $\chi^{2}=\mathbf{1}$ by (ii).)

Proof. The reducibility of the following generalized principal series $\nu^{s} \chi \rtimes \mathbf{1}(s \in \mathbb{R}$, $\chi$ unitary and unramified) is well known in the connected case. For $O(2, F)$ we use the simple Mackey machinery to verify the result since in that case we consider $\operatorname{Ind}_{S O(2, F)}^{O(2, F)}(\chi)$ and $S O(2, F) \simeq F^{\times}$.

\section{The Case of Classical groups}

We begin this section with a reformulation of the basic result of Satake (cf. Car. for connected case and [Ra] for a split even-orthogonal group).

Theorem 4.1. (i) Assume that $\left(\chi_{1}, \ldots, \chi_{n}\right)$ is a sequence of unramified characters of $F^{\times}$. Then the induced representation $\left(\right.$of $\left.G_{n}\right) \chi_{1} \times \cdots \times \chi_{n} \rtimes \mathbf{1}$ contains the unique unramified irreducible subquotient. We write $\sigma_{\left(\chi_{1}, \ldots, \chi_{n}\right)}$.

(ii) Assume that $\left(\chi_{1}, \ldots, \chi_{n}\right)$ and $\left(\chi_{1}^{\prime}, \ldots, \chi_{n}^{\prime}\right)$ are two sequences of unramified characters of $F^{\times}$. Then $\sigma_{\left(\chi_{1}, \ldots, \chi_{n}\right)} \simeq \sigma_{\left(\chi_{1}^{\prime}, \ldots, \chi_{n}^{\prime}\right)}$ if and only if there is a permutation $\alpha$ of $n$-letters and a sequence $\left(\epsilon_{1}, \ldots, \epsilon_{n}\right) \in\{ \pm 1\}^{n}$ such that $\chi_{i}^{\prime}=\chi_{\alpha(i)}^{\epsilon_{i}}, i=1, \ldots, n$.

(iii) Assume that $\sigma \in \operatorname{Irr} G_{n}$ is an unramified representation. Then there exists a sequence $\left(\chi_{1}, \ldots, \chi_{n}\right)$ of unramified characters of $F^{\times}$such that $\sigma \simeq$ $\sigma_{\left(\chi_{1}, \ldots, \chi_{n}\right)}$. Any such sequence we call a supercuspidal support of $\sigma$.

Corollary 4.1. Assume that $\sigma \in \operatorname{Irr} G_{n}$ is an unramified representation. Then $\sigma$ is self-dual: $\sigma \simeq \widetilde{\sigma}$. 
We present the following two definitions that are fundamental for us.

Definition 4.1. Assume that $\sigma \in \operatorname{Irr} G_{n}$ is an unramified representation. We say that $\sigma$ is strongly negative if for any constituent $\chi_{1} \otimes \chi_{2} \otimes \cdots \otimes \chi_{n}$ of $\operatorname{Jacq}_{(1, \ldots, 1 ; 0)}(\sigma)$ we have

$$
\left\{\begin{array}{l}
e\left(\chi_{1}\right)<0 \\
e\left(\chi_{1}\right)+e\left(\chi_{2}\right)<0, \\
\cdots \\
e\left(\chi_{1}\right)+e\left(\chi_{2}\right)+\cdots+e\left(\chi_{n}\right)<0 .
\end{array}\right.
$$

Definition 4.2. Assume that $\sigma \in \operatorname{Irr} G_{n}$ is an unramified representation. We say that $\sigma$ is negative if for any constituent $\chi_{1} \otimes \chi_{2} \otimes \cdots \otimes \chi_{n}$ of $\operatorname{Jacq}_{(1, \ldots, 1 ; 0)}(\sigma)$ we have

$$
\left\{\begin{array}{l}
e\left(\chi_{1}\right) \leq 0 \\
e\left(\chi_{1}\right)+e\left(\chi_{2}\right) \leq 0, \\
\cdots \\
e\left(\chi_{1}\right)+e\left(\chi_{2}\right)+\cdots+e\left(\chi_{n}\right) \leq 0 .
\end{array}\right.
$$

We continue computing some Jacquet module multiplicities. We start with the next lemma.

Lemma 4.1. Assume that $\chi$ is an unramified unitary character of $F^{\times}, \alpha, \beta \in \mathbb{R}$, $\alpha+\beta+1 \in \mathbb{Z}_{>0}$, and $\sigma \in \operatorname{Irr} G_{n}$ an unramified representation. If $\mu^{*}(\sigma) \geq$ $\zeta(-\beta, \alpha, \chi) \otimes \sigma^{\prime \prime}, \sigma^{\prime \prime} \in \operatorname{Irr} G_{n-\alpha-\beta-1}$, then there is a unique unramified representation $\sigma^{\prime} \in \operatorname{Irr} G_{n-\alpha-\beta-1}$ such that $\mu^{*}(\sigma) \geq \zeta(-\beta, \alpha, \chi) \otimes \sigma^{\prime}$. Moreover, if such an unramified representation exists, it must share a supercuspidal support with $\sigma^{\prime \prime}$ and we must have

$$
\sigma \hookrightarrow \zeta(-\beta, \alpha, \chi) \rtimes \sigma^{\prime} .
$$

Proof. First, $\mu^{*}(\sigma) \geq \zeta(-\beta, \alpha, \chi) \otimes \sigma^{\prime \prime}$ implies that

$$
\operatorname{Jacq}_{(1, \ldots, 1 ; n-\alpha-\beta-1)}(\sigma) \geq \chi \nu^{-\beta} \otimes \chi \nu^{-\beta+1} \otimes \cdots \otimes \chi \nu^{\alpha} \otimes \sigma^{\prime \prime} .
$$

Thus, there exists an irreducible representation $\sigma^{\prime}$ such that

$$
\operatorname{Jacq}_{(1, \ldots, 1 ; n-\alpha-\beta-1)}(\sigma) \rightarrow \chi \nu^{-\beta} \otimes \chi \nu^{-\beta+1} \otimes \cdots \otimes \chi \nu^{\alpha} \otimes \sigma^{\prime} .
$$

Clearly, the transitivity of Jacquet modules implies that $\sigma^{\prime}$ and $\sigma^{\prime \prime}$ share the supercuspidal support. Moreover, (4.3) and the Frobenius reciprocity implies

$$
\sigma \hookrightarrow \chi \nu^{-\beta} \times \chi \nu^{-\beta+1} \times \cdots \times \chi \nu^{\alpha} \rtimes \sigma^{\prime} .
$$

Now, (4.4) and Lemma 1.1(ii) imply that $\sigma^{\prime}$ is unramified. Moreover, (4.4) and Theorem 4.1 imply its uniqueness. Also, inducing in stages, we see that

$$
\zeta(-\beta, \alpha, \chi) \rtimes \sigma^{\prime} \hookrightarrow \chi \nu^{-\beta} \times \chi \nu^{-\beta+1} \times \cdots \times \chi \nu^{\alpha} \rtimes \sigma^{\prime} .
$$

Now, Lemma 1.1(i), Theorem 4.1(i) and (4.4) complete the proof.

Now, we compute some Jacquet modules. 
Lemma 4.2. Assume that $\chi$ is an unramified unitary character of $F^{\times}, \alpha, \beta \in \mathbb{R}$, $\alpha+\beta+1 \in \mathbb{Z}_{>0}$, and $\sigma \in \operatorname{Irr} G_{n}$ an unramified representation. Then if $\chi^{2} \neq \mathbf{1}$ or $\beta \leq \alpha$, we have the the following formula:

$$
\begin{aligned}
& \operatorname{Mult}\left(\zeta(-\beta, \alpha, \chi) \otimes \sigma, \mu^{*}\left(\zeta\left(-\alpha, \beta, \chi^{-1}\right) \rtimes \sigma\right)\right) \\
& =\operatorname{Mult}\left(\zeta(-\beta, \alpha, \chi) \otimes \sigma, \mu^{*}(\zeta(-\beta, \alpha, \chi) \rtimes \sigma)\right) \\
& \quad=\kappa\left(1+\sum_{j=0}^{\alpha+\beta} \operatorname{Mult}\left(\zeta(j-\beta, \alpha, \chi) \otimes \sigma_{j}, \mu^{*}(\sigma)\right)\right),
\end{aligned}
$$

where

$$
\kappa= \begin{cases}1, & \alpha \neq \beta \\ 2, & \alpha=\beta\end{cases}
$$

and $\sigma_{j}$ is the unique (if exists) unramified representation characterized by

$$
\operatorname{Jacq}_{(\alpha+\beta-j+1 ; n-\alpha-\beta-1+j)}(\sigma) \geq \zeta(j-\beta, \alpha, \chi) \otimes \sigma_{j} .
$$

Proof. We analyse $\zeta(-\beta, \alpha, \chi) \otimes \sigma \leq \mu^{*}\left(\zeta\left(-\alpha, \beta, \chi^{-1}\right) \rtimes \sigma\right)$ using Theorem 3.1. Thus, we take indices $0 \leq j \leq i \leq \alpha+\beta+1$ and an irreducible constituent $\mu^{*}(\sigma) \geq \zeta \otimes \sigma^{\prime}$ such that

$$
\left\{\begin{array}{l}
\zeta(-\beta, \alpha, \chi) \leq \zeta\left(-\alpha, \beta-i, \chi^{-1}\right) \times \zeta(-\beta, j-\beta-1, \chi) \times \zeta \\
\sigma \leq \zeta(j-\beta, i-\beta-1, \chi) \rtimes \sigma^{\prime} .
\end{array}\right.
$$

The first inequality shows that if $\chi^{2} \neq \mathbf{1}$ or $\alpha>\beta$, we must have $i=\alpha+\beta+1$ and

$$
\left\{\begin{array}{l}
\zeta(-\beta, \alpha, \chi) \leq \zeta(-\beta, j-\beta-1, \chi) \times \zeta, \\
\sigma \leq \zeta(j-\beta, \alpha, \chi) \rtimes \sigma^{\prime} .
\end{array}\right.
$$

One can now complete the proof of the lemma combining Lemma 1.1 and Lemma 4.1. The remaining case is treated similarly.

The next lemma is an important but simple technical result.

Lemma 4.3. Assume that $\sigma \in \operatorname{Irr} G_{n}$ is an unramified representation. Next, assume that $\pi_{i} \in \operatorname{Irr} G L\left(n_{i}, F\right), i=1, \ldots, l$, are unramified representations. Let $\pi$ be the unique unramified irreducible subquotient of $\pi_{1} \times \cdots \times \pi_{l}$. Let $\sigma^{\prime} \in \operatorname{Irr} G_{n^{\prime}}$ be an unramified representation. Now, if $\sigma \hookrightarrow \pi_{1} \times \cdots \times \pi_{l} \rtimes \sigma^{\prime}$, then $\sigma \hookrightarrow \pi \rtimes \sigma^{\prime}$.

Proof. Let $\Pi$ be the subrepresentation of $\pi_{1} \times \cdots \times \pi_{l}$ such that

$$
\pi \hookrightarrow\left(\pi_{1} \times \cdots \times \pi_{l}\right) / \Pi .
$$

We get $\Pi$ considering the Jordan-Hoelder series of $\pi_{1} \times \cdots \times \pi_{l}$. Next, since the functor of induction is exact in the category of all smooth admissible representations of finite length, we see that either the composition of the next equivariant morphism is non-zero:

$$
\sigma \hookrightarrow \pi_{1} \times \cdots \times \pi_{l} \rtimes \sigma^{\prime} \rightarrow\left(\pi_{1} \times \cdots \times \pi_{l} \rtimes \sigma^{\prime}\right) /\left(\Pi \rtimes \sigma^{\prime}\right)
$$

or

$$
\sigma \hookrightarrow \Pi \rtimes \sigma^{\prime} .
$$

The latter is not possible by Lemma 1.1(ii) and the definition of $\Pi$. Thus, we see that both $\sigma$ and $\pi \rtimes \sigma^{\prime}$ embed into $\left(\pi_{1} \times \cdots \times \pi_{l} \rtimes \sigma^{\prime}\right) /\left(\Pi \rtimes \sigma^{\prime}\right)$. 
Lemma 4.4. Assume that $\sigma \in \operatorname{Irr} G_{n}$ is an unramified representation. Moreover, assume that $\chi$ is an unramified unitary character of $F^{\times}, \alpha, \beta \in \mathbb{R}, \alpha+\beta+1 \in$ $\mathbb{Z}_{>0}$, and $\sigma^{\prime} \in \operatorname{Irr} G_{n^{\prime}}$ is an unramified representation. Assume that $\alpha-\beta>0$ and $\mu^{*}(\sigma) \geq \zeta(-\beta, \alpha, \chi) \otimes \sigma^{\prime}$ such that $\alpha-\beta$ is the largest possible value. Then $\sigma \hookrightarrow \zeta(-\beta, \alpha, \chi) \rtimes \sigma^{\prime}$ is a unique irreducible subrepresentation.

Proof. Lemma 4.1 shows that $\sigma \hookrightarrow \zeta(-\beta, \alpha, \chi) \rtimes \sigma^{\prime}$. Next, Lemma 4.2 shows that $\zeta(-\beta, \alpha, \chi) \otimes \sigma^{\prime}$ appears in $\mu^{*}\left(\zeta(-\beta, \alpha, \chi) \otimes \sigma^{\prime}\right)$ with multiplicity one unless there exists $0 \leq j \leq \alpha+\beta$, and unramified representation $\sigma_{j}$ such that

$$
\sigma^{\prime} \hookrightarrow \zeta(-\beta+j, \alpha, \chi) \rtimes \sigma_{j} .
$$

Hence we see that

$$
\begin{aligned}
\sigma \hookrightarrow \zeta(-\beta, \alpha, \chi) \rtimes \sigma^{\prime} \hookrightarrow \zeta(-\beta, \alpha, \chi) \times \zeta( & -\beta+j, \alpha, \chi) \rtimes \sigma_{j}, \\
& \simeq \zeta(-\beta+j, \alpha, \chi) \times \zeta(-\beta, \alpha, \chi) \rtimes \sigma_{j} .
\end{aligned}
$$

Now, Lemma 4.1 shows that for some unramified representation $\sigma_{1}^{\prime}$ we have

$$
\sigma \hookrightarrow \zeta(-\beta+j, \alpha, \chi) \rtimes \sigma_{1}^{\prime} .
$$

This contradicts our assumption unless $j=0$. Now, continuing this process after finitely many steps we find an unramified representation $\sigma^{\prime \prime}$ such that $\mu^{*}\left(\sigma^{\prime \prime}\right) \nsupseteq$ $\zeta(-\beta, \alpha, \chi) \otimes \sigma_{1}^{\prime}$, for any irreducible representation $\sigma_{1}^{\prime}$, and

$$
\left\{\begin{array}{l}
\sigma \hookrightarrow \zeta_{m} \rtimes \sigma^{\prime \prime}, \\
\sigma^{\prime} \hookrightarrow \zeta_{m-1} \rtimes \sigma^{\prime \prime}
\end{array}\right.
$$

where

$$
\zeta_{m}:=\zeta(-\beta, \alpha, \chi) \times \cdots \times \zeta(-\beta, \alpha, \chi) \quad(m \text { factors })
$$

is an irreducible unramified representation (cf. [Ze]). We show that $\zeta_{m} \otimes \sigma^{\prime \prime}$ appears in $\mu^{*}\left(\zeta_{m} \rtimes \sigma^{\prime \prime}\right)$ with multiplicity one. This will prove the lemma. The assumption on $\sigma^{\prime \prime}$ can be stated as follows.

Claim 1. Under the above assumptions, $\mu^{*}\left(\sigma^{\prime \prime}\right) \nsupseteq \zeta(-\beta+j, \alpha, \chi) \otimes \sigma_{1}^{\prime}$, for any irreducible representation $\sigma_{1}^{\prime}, 0 \leq j \leq \alpha+\beta$.

Proof. The case $j=0$ is our assumption on $\sigma^{\prime \prime}$. The case $j>0$ proceeds the same way as the similar case for $\sigma^{\prime}$ above.

Now, we are ready to check the desired multiplicity. Applying Theorem $3.1 \mathrm{~m}$ times we find indices $0 \leq j_{a} \leq i_{a} \leq \alpha+\beta+1,1 \leq a \leq m$, and an irreducible representation $\mu^{*}\left(\sigma^{\prime \prime}\right) \geq \zeta \otimes \sigma_{1}^{\prime}$ such that

$$
\zeta_{m} \leq \zeta \times \prod_{a=1}^{m} \zeta\left(-\alpha, \beta-i_{a}, \chi^{-1}\right) \times \zeta\left(-\beta, j_{a}-\beta-1, \chi\right)
$$

and

$$
\sigma^{\prime \prime} \leq \prod_{a=1}^{m} \zeta\left(j_{a}-\beta, i_{a}-\beta-1, \chi\right) \rtimes \sigma_{1}^{\prime} .
$$

Since $-\beta>-\alpha$, we see that (4.5) implies $i_{a}=\alpha+\beta+1$, for all $a$. Thus, (4.5) is in fact

$$
\zeta_{m} \leq \zeta \times \prod_{a=1}^{m} \zeta\left(-\beta, j_{a}-\beta-1, \chi\right)
$$


Since on the left-hand side of (4.7) the term $\chi \nu^{\alpha}$ appears exactly $m$ times, the right-hand side must satisfy the same. Since Claim 1 implies that $\chi \nu^{\alpha}$ cannot come from $\zeta$, we see that each $\zeta\left(-\beta, j_{a}-\beta-1, \chi\right)$ must produce that term. Thus $j_{a}=\alpha+\beta+1$, for all $a$. This implies that $\zeta$ is trivial and thus $\sigma^{\prime \prime} \cong \sigma_{1}^{\prime}$.

Lemma 4.5. Assume that $\sigma \in \operatorname{Irr} G_{n}$ is an unramified representation. Then we have

$$
\sigma \hookrightarrow \zeta\left(-\beta_{1}, \alpha_{1}, \chi_{1}\right) \times \cdots \times \zeta\left(-\beta_{k}, \alpha_{k}, \chi_{k}\right) \rtimes \sigma_{n e g},
$$

where the data are as follows:

- the sequence of representations $\zeta\left(-\beta_{i}, \alpha_{i}, \chi_{i}\right)\left(\alpha_{i}, \beta_{i} \in \mathbb{R}, \alpha_{i}+\beta_{i} \in \mathbb{Z}_{\geq 0}, \chi_{i}\right.$ is an unramified unitary character of $\left.F^{\times}\right), 1 \leq i \leq k$, is such that $\alpha_{i}-\beta_{i} \geq \alpha_{j}-\beta_{j}$ whenever $\chi_{i}=\chi_{j}$ and $\alpha_{i}-\beta_{i}>0$ for all $i \leq j$,

- $\sigma_{n e g}$ is a negative unramified representation.

Proof. If $\sigma$ is negative, then $\sigma \simeq \sigma_{n e g}$ and we are done. Thus assume that $\sigma$ is not negative. Then, we can find unramified characters $\chi_{1}, \ldots, \chi_{n}$ such that

$$
\operatorname{Jacq}_{(1, \ldots, 1 ; 0)}(\sigma) \geq \chi_{1} \otimes \cdots \otimes \chi_{n},
$$

and $1 \leq k \leq n$ such that

$$
e\left(\chi_{1}\right)+\cdots+e\left(\chi_{k}\right)>0 .
$$

In fact, we have

$$
\operatorname{Jacq}_{(1, \ldots, 1 ; 0)}(\sigma) \rightarrow \chi_{1} \otimes \cdots \otimes \chi_{n}
$$

which is equivalent to

$$
\sigma \hookrightarrow \chi_{1} \times \cdots \times \chi_{n} \rtimes \mathbf{1} .
$$

Now, we partition the set of indices $\{1, \ldots, k\}$. We say that $i$ and $j$ are equivalent if $\chi_{i}^{u}=\chi_{j}^{u}$. This is clearly a relation of equivalence on $\{1, \ldots, k\}$. Thus, for each of its partitions $\mathcal{J}$ we cannot have

$$
\sum_{i \in \mathcal{J}} e\left(\chi_{i}\right) \leq 0
$$

or summing over all partitions would imply $e\left(\chi_{1}\right)+\cdots+e\left(\chi_{k}\right) \leq 0$. This contradicts (4.8). Now, we fix a partition $\mathcal{J}$ such that

$$
\sum_{i \in \mathcal{J}} e\left(\chi_{i}\right)>0 \text {. }
$$

Let us write $\mathcal{J}=\left\{i_{1}, \ldots, i_{l}\right\}, i_{1}<i_{2}<\cdots<i_{l}$. Now, since [Ze] or Lemma 2.1 here imply that

$$
\chi_{i} \times \chi_{j} \cong \chi_{j} \times \chi_{i}, \quad i \in \mathcal{J}, j \notin \mathcal{J}, 1 \leq j \leq k,
$$

we can use (4.9) to write

$$
\sigma \hookrightarrow \chi_{i_{1}} \times \cdots \times \chi_{i_{l}} \times \text { (other characters in the same order) } \rtimes \mathbf{1} \text {. }
$$

This can be rewritten using Lemma 4.3 as follows:

$$
\sigma \hookrightarrow \pi \times \text { (other characters in the same order) } \rtimes \mathbf{1},
$$

where $\pi$ is the unique unramified irreducible subquotient of $\chi_{i_{1}} \times \cdots \times \chi_{i_{l}}$. Now, we apply Lemma 2.1 and Theorem 2.1 to write

$$
\pi \simeq \zeta\left(-\beta_{1}^{\prime}, \alpha_{1}^{\prime}, \chi_{1}^{\prime}\right) \times \cdots \times \zeta\left(-\beta_{k^{\prime}}^{\prime}, \alpha_{k^{\prime}}^{\prime}, \chi_{k^{\prime}}^{\prime}\right),
$$


where $\alpha_{i}^{\prime}, \beta_{i}^{\prime} \in \mathbb{R}$ and $\chi_{i}^{\prime}$ is a unitary unramified character, $i=1, \ldots, k$. Comparing central characters and using (4.10), we see that $\alpha_{i_{0}}^{\prime}-\beta_{i_{0}}^{\prime}>0$ for some $i_{0}$. Combining (4.11) and (4.12) we see that

$$
\sigma \hookrightarrow \zeta\left(-\beta_{i_{0}}^{\prime}, \alpha_{i_{0}}^{\prime}, \chi_{i_{0}}^{\prime}\right) \times \cdots .
$$

Now, we can apply the previous lemma and induction. Thus, there exists an embedding

$$
\sigma \hookrightarrow \zeta\left(-\beta_{1}, \alpha_{1}, \chi_{1}\right) \times \cdots \times \zeta\left(-\beta_{k}, \alpha_{k}, \chi_{k}\right) \rtimes \sigma_{n e g},
$$

where inducing data is as in the statement of the lemma.

The induced representation mentioned in the statement of the Lemma 4.5 is not unique. A bit more work is needed to get the uniqueness. First, we have the following lemma.

Lemma 4.6. Assume that the representations $\zeta\left(-\beta_{i}, \alpha_{i}, \chi_{i}\right)\left(\alpha_{i}, \beta_{i} \in \mathbb{R}, \alpha_{i}+\beta_{i} \in\right.$ $\mathbb{Z}_{\geq 0}, \chi_{i}$ is an unramified unitary character of $\left.F^{\times}\right), 1 \leq i \leq k$, satisfy conditions of Lemma 4.5. Then its unique unramified subquotient is of the form $\zeta\left(-\beta_{1}^{\prime}, \alpha_{1}^{\prime}, \chi_{1}^{\prime}\right) \times$ $\cdots \times \zeta\left(-\beta_{k^{\prime}}^{\prime}, \alpha_{k^{\prime}}^{\prime}, \chi_{k^{\prime}}^{\prime}\right)\left(\alpha_{i}^{\prime}, \beta_{i}^{\prime} \in \mathbb{R}, \alpha_{i}^{\prime}+\beta_{i}^{\prime} \in \mathbb{Z}_{\geq 0}, \chi_{i}^{\prime}\right.$ is an unramified unitary character of $\left.F^{\times}\right)$, where

$$
\alpha_{i}^{\prime}-\beta_{i}^{\prime}>0, \quad 1 \leq i \leq k^{\prime} .
$$

Proof. Let us take $i<j$. Then we have two cases. First, we consider $\alpha_{j} \geq \alpha_{i}$. Then since $\alpha_{i}-\beta_{i} \geq \alpha_{j}-\beta_{j}$ we have $0 \geq \alpha_{i}-\alpha_{j} \geq \beta_{i}-\beta_{j}$. Thus if $\chi_{i}=\chi_{j}$ and $\alpha_{i}-\alpha_{j} \in \mathbb{Z}$, then $\zeta\left(-\beta_{i}, \alpha_{i}, \chi_{i}\right) \times \zeta\left(-\beta_{j}, \alpha_{j}, \chi_{j}\right)$ is irreducible since $\alpha_{j} \geq \alpha_{i} \geq-\beta_{i} \geq-\beta_{j}$. Next, we consider $\alpha_{i}>\alpha_{j}$. Then if $\zeta\left(-\beta_{i}, \alpha_{i}, \chi_{i}\right) \times \zeta\left(-\beta_{j}, \alpha_{j}, \chi_{j}\right)$ is reducible, we have $\chi_{i}=\chi_{j}$, and

$$
\zeta\left(-\beta_{i}, \alpha_{i}, \chi_{i}\right) \times \zeta\left(-\beta_{j}, \alpha_{j}, \chi_{j}\right) \rightarrow \zeta\left(-\beta_{j}, \alpha_{i}, \chi_{i}\right) \times \zeta\left(-\beta_{i}, \alpha_{j}, \chi_{j}\right) .
$$

Note that in that case we must have $\alpha_{i}>\alpha_{j} \geq-\beta_{i}>-\beta_{j}$ or $\alpha_{i}>\alpha_{j}=-\beta_{i}-1>$ $-\beta_{j}$. In both cases $\alpha_{i}-\beta_{j} \geq \alpha_{j}-\beta_{j}>0$. In the first case, $\alpha_{j}-\beta_{i}>\alpha_{j}-\beta_{j}>0$. Thus the process of "linking" (cf. [Ze]) preserves the positivity.

The next lemma represents the first classification result for unramified representations.

Lemma 4.7. Assume that $\sigma \in \operatorname{Irr} G_{n}$ is an unramified representation. Then $\sigma$ is a unique irreducible subrepresentation

$$
\sigma \hookrightarrow \zeta\left(-\beta_{1}, \alpha_{1}, \chi_{1}\right) \times \cdots \times \zeta\left(-\beta_{k}, \alpha_{k}, \chi_{k}\right) \rtimes \sigma_{n e g},
$$

where:

- the (unique up to permutation) sequence of representations $\zeta\left(-\beta_{i}, \alpha_{i}, \chi_{i}\right)\left(\alpha_{i}, \beta_{i}\right.$ $\in \mathbb{R}, \alpha_{i}+\beta_{i} \in \mathbb{Z}_{\geq 0}, \chi_{i}$ is an unramified unitary character of $\left.F^{\times}\right), 1 \leq i \leq k$, is such that $\zeta\left(-\beta_{1}, \alpha_{1}, \chi_{1}\right) \times \cdots \times \zeta\left(-\beta_{k}, \alpha_{k}, \chi_{k}\right)$ is irreducible and $\alpha_{i}-\beta_{i}>0$ for all $i$,

- $\sigma_{n e g}$ is a (unique) negative unramified representation.

Proof. The existence of an induced representation such that (4.13) holds is a direct consequence of Lemma 4.5, Lemma 4.6 and Lemma 4.3. We note that since $\zeta\left(-\beta_{1}, \alpha_{1}, \chi_{1}\right) \times \cdots \times \zeta\left(-\beta_{k}, \alpha_{k}, \chi_{k}\right)$ is irreducible we can permute $\zeta$ 's as we want; 
it does not affect (4.13). In particular, we may assume $\alpha_{1} \geq \alpha_{2} \geq \cdots \geq \alpha_{k}$. Refining an argument used in the proof of Lemma 4.4 a little bit we can show that $\zeta\left(-\beta_{1}, \alpha_{1}, \chi_{1}\right) \times \cdots \times \zeta\left(-\beta_{k}, \alpha_{k}, \chi_{k}\right) \otimes \sigma_{n e g}$ appears in

$$
\mu^{*}\left(\zeta\left(-\beta_{1}, \alpha_{1}, \chi_{1}\right) \times \cdots \times \zeta\left(-\beta_{k}, \alpha_{k}, \chi_{k}\right) \rtimes \sigma_{n e g}\right)
$$

with multiplicity one. We leave details to the reader.

Now, we investigate the uniqueness of the inducing data. So, assume further that $\sigma$ is a unique irreducible subrepresentation:

$$
\sigma \hookrightarrow \zeta\left(-\beta_{1}^{\prime}, \alpha_{1}^{\prime}, \chi_{1}^{\prime}\right) \times \cdots \times \zeta\left(-\beta_{k^{\prime}}^{\prime}, \alpha_{k^{\prime}}^{\prime}, \chi_{k^{\prime}}^{\prime}\right) \rtimes \sigma_{n e g}^{\prime},
$$

where:

- the sequence of representations $\zeta\left(-\beta_{i}^{\prime}, \alpha_{i}^{\prime}, \chi_{i}^{\prime}\right)\left(\alpha_{i}^{\prime}, \beta_{i}^{\prime} \in \mathbb{R}, \alpha_{i}^{\prime}+\beta_{i}^{\prime} \in \mathbb{Z}_{\geq 0}, \chi_{i}^{\prime}\right.$ is an unramified unitary character of $\left.F^{\times}\right), 1 \leq i \leq k^{\prime}$, is such that $\zeta\left(-\beta_{1}^{\prime}, \alpha_{1}^{\prime}, \chi_{1}^{\prime}\right) \times$ $\cdots \times \zeta\left(-\beta_{k}^{\prime}, \alpha_{k}^{\prime}, \chi_{k}\right)$ is irreducible and $\alpha_{i}^{\prime}-\beta_{i}^{\prime}>0$ for all $i$,

- $\sigma_{\text {neg }}^{\prime}$ is a negative unramified representation.

We also assume

$$
\left\{\begin{array}{l}
\alpha_{1} \geq \alpha_{2} \geq \cdots \geq \alpha_{k} \\
\alpha_{1}^{\prime} \geq \alpha_{2}^{\prime} \geq \cdots \geq \alpha_{k^{\prime}}^{\prime}
\end{array}\right.
$$

This can be combined with our assumption that $\zeta\left(-\beta_{i}, \alpha_{i}, \chi_{i}\right) \times \zeta\left(-\beta_{j}, \alpha_{j}, \chi_{j}\right)$ and $\zeta\left(-\beta_{i}^{\prime}, \alpha_{i}^{\prime}, \chi_{i}^{\prime}\right) \times \zeta\left(-\beta_{j}^{\prime}, \alpha_{j}^{\prime}, \chi_{j}^{\prime}\right)$ are irreducible to assume the following $(i<j)$ :

$$
\left\{\begin{array}{l}
\alpha_{i} \geq \alpha_{j}>-\beta_{j} \geq-\beta_{i} \\
\alpha_{i} \geq-\beta_{i}>\alpha_{j}+1>\alpha_{j} \geq-\beta_{j}
\end{array}\right.
$$

and similarly

$$
\left\{\begin{array}{l}
\alpha_{i}^{\prime} \geq \alpha_{j}^{\prime} \geq-\beta_{j}^{\prime} \geq-\beta_{i}^{\prime}, \\
\alpha_{i}^{\prime}>-\beta_{i}^{\prime}>\alpha_{j}^{\prime}+1>\alpha_{j}^{\prime}>-\beta_{j}^{\prime},
\end{array}\right.
$$

We first investigate the following obvious consequences of (4.13) and (4.14):

$$
\begin{aligned}
\mu^{*}\left(\zeta\left(-\beta_{1}, \alpha_{1}, \chi_{1}\right) \times \cdots\right. & \left.\times \zeta\left(-\beta_{k}, \alpha_{k}, \chi_{k}\right) \rtimes \sigma_{n e g}\right) \\
& \geq \zeta\left(-\beta_{1}^{\prime}, \alpha_{1}^{\prime}, \chi_{1}^{\prime}\right) \times \cdots \times \zeta\left(-\beta_{k^{\prime}}^{\prime}, \alpha_{k^{\prime}}^{\prime}, \chi_{k^{\prime}}^{\prime}\right) \otimes \sigma_{n e g}^{\prime}
\end{aligned}
$$

and

$$
\begin{aligned}
\mu^{*}\left(\zeta\left(-\beta_{1}^{\prime}, \alpha_{1}^{\prime}, \chi_{1}^{\prime}\right) \times \cdots \times \zeta\left(-\beta_{k^{\prime}}^{\prime}, \alpha_{k^{\prime}}^{\prime}, \chi_{k^{\prime}}^{\prime}\right) \rtimes \sigma_{n e g}^{\prime}\right) & \\
& \geq \zeta\left(-\beta_{1}, \alpha_{1}, \chi_{1}\right) \times \cdots \times \zeta\left(-\beta_{k}, \alpha_{k}, \chi_{k}\right) \otimes \sigma_{n e g}
\end{aligned}
$$

Applying Theorem $3.1 k$ times (resp. $k^{\prime}$ times) we find indices $0 \leq j_{a} \leq i_{a} \leq \alpha_{a}+$ $\beta_{a}+1,0 \leq j_{a}^{\prime} \leq i_{a} \leq \alpha_{a}^{\prime}+\beta_{a}^{\prime}+1$, and irreducible representations $\mu^{*}\left(\sigma_{\text {neg }}\right) \geq \zeta \otimes \sigma_{1}$ 
and $\mu^{*}\left(\sigma_{\text {neg }}^{\prime}\right) \geq \zeta^{\prime} \otimes \sigma_{1}^{\prime}$ such that

$$
\begin{aligned}
\zeta\left(-\beta_{1}^{\prime}, \alpha_{1}^{\prime}, \chi_{1}^{\prime}\right) \times \cdots \times \zeta\left(-\beta_{k^{\prime}}^{\prime}, \alpha_{k^{\prime}}^{\prime}, \chi_{k^{\prime}}^{\prime}\right) \\
\leq \zeta \times \prod_{a=1}^{k} \zeta\left(-\alpha_{a}, \beta_{a}-i_{a}, \chi_{a}^{-1}\right) \times \zeta\left(-\beta_{a}, j_{a}-\beta_{a}-1, \chi_{a}\right), \\
\zeta\left(-\beta_{1}, \alpha_{1}, \chi_{1}\right) \times \cdots \times \zeta\left(-\beta_{k}, \alpha_{k}, \chi_{k}\right) \\
\leq \zeta^{\prime} \times \prod_{a=1}^{k^{\prime}} \zeta\left(-\alpha_{a}^{\prime}, \beta_{a}^{\prime}-i_{a}^{\prime}, \chi_{a}^{\prime}-1\right) \times \zeta\left(-\beta_{a}^{\prime}, j_{a}^{\prime}-\beta_{a}^{\prime}-1, \chi_{a}^{\prime}\right), \\
\sigma_{n e g}^{\prime} \leq \prod_{a=1}^{k} \zeta\left(j_{a}-\beta_{a}, i_{a}-\beta_{a}-1, \chi_{a}\right) \rtimes \sigma_{1},
\end{aligned}
$$

and

$$
\sigma_{n e g} \leq \prod_{a=1}^{k^{\prime}} \zeta\left(j_{a}^{\prime}-\beta_{a}^{\prime}, i_{a}^{\prime}-\beta_{a}^{\prime}-1, \chi_{a}^{\prime}\right) \rtimes \sigma_{1}^{\prime} .
$$

We first show that $\alpha_{1}=\alpha_{1}^{\prime}$. If not, then $\alpha_{1}^{\prime}>\alpha_{1}$ or $\alpha_{1}>\alpha_{1}^{\prime}$. Now, $\alpha_{1}^{\prime}>\alpha_{1}$ and (4.20) show that $\zeta \simeq \zeta\left(-l, \alpha_{1}^{\prime}, \chi_{1}^{\prime}\right) \times \cdots$, where $l$ must be one of the exponents on the left-hand side of (4.20). Since $\alpha_{1}^{\prime} \geq \alpha_{i}^{\prime}>\beta_{i}^{\prime}$, we see that $\alpha_{i}^{\prime} \geq-\beta_{i}^{\prime}>-\alpha_{1}^{\prime}$. Thus, we see that $l>-\alpha_{1}^{\prime}$. This contradicts the fact that $\sigma_{\text {neg }}$ is negative. Similarly we show that the number of indices $i$ such that $\alpha_{1}^{\prime}=\alpha_{i}^{\prime}$ is equal to the number of indices $i$ such that $\alpha_{1}=\alpha_{i}$. Let $l$ denote the number of such indices. Then clearly, $\chi_{a}^{\prime}=\chi_{a}, i_{a}=j_{a}=\alpha_{a}+\beta_{a}+1, i_{a}^{\prime}=j_{a}^{\prime}=\alpha_{a}^{\prime}+\beta_{a}^{\prime}+1, a=1, \ldots, l$, in (4.20) and (4.21). Next, (4.16), (4.17) and Zelevinsky theory (that is, the way we get left-hand sides from right-hand sides in (4.20) and (4.21)) imply that $\beta_{1}, \ldots, \beta_{l}$ must be up to the permutation equal to $\beta_{1}^{\prime}, \ldots, \beta_{l}^{\prime}$. Now, (4.16) and (4.17) imply that $\beta_{a}=\beta_{a}^{\prime}$, $a=1, \ldots, l$. This can be continued, finally proving the uniqueness of the data. We should remark that if $k^{\prime}>k$ or $k>k^{\prime}$, some of the terms of the left-hand sides of (4.20) and (4.21), respectively, are not obtained by this procedure. Thus, they must be produced by either $\zeta$ or $\zeta^{\prime}$. This is a contradiction, arguing as in the proof of $\alpha_{1}=\alpha_{1}^{\prime}$.

Lemma 4.8. Assume that the data is given as follows:

- the sequence of representations $\zeta\left(-\beta_{i}, \alpha_{i}, \chi_{i}\right)\left(\alpha_{i}, \beta_{i} \in \mathbb{R}, \alpha_{i}+\beta_{i} \in \mathbb{Z}_{>0}, \chi_{i}\right.$ is an unramified unitary character of $\left.F^{\times}\right), 1 \leq i \leq k$, is such that $\zeta\left(-\beta_{1}, \alpha_{1}, \chi_{1}\right) \times$ $\cdots \times \zeta\left(-\beta_{k}, \alpha_{k}, \chi_{k}\right)$ is irreducible and $\alpha_{i}-\beta_{i}>0$, for all $i$,

- $\sigma_{n e g}$ is a negative unramified representation.

Then

$$
\zeta\left(-\beta_{1}, \alpha_{1}, \chi_{1}\right) \times \cdots \times \zeta\left(-\beta_{k}, \alpha_{k}, \chi_{k}\right) \rtimes \sigma_{n e g}
$$

has a unique irreducible subrepresentation, and that representation appears with multiplicity one in its composition series. This representation is self-dual. Moreover, this induced representation is irreducible if and only if $\zeta\left(-\beta_{i}, \alpha_{i}, \chi_{i}\right) \times$ $\zeta\left(-\alpha_{j}, \beta_{j}, \chi_{j}^{-1}\right), i \neq j$, and $\zeta\left(-\beta_{i}, \alpha_{i}, \chi_{i}\right) \rtimes \sigma_{n e g}, i, j=1, \ldots, k$, are irreducible.

Proof. The uniqueness of irreducible subrepresentation and the fact that it appears with multiplicity one in its composition series is proved as in the previous lemma. Now, we put $\zeta_{i}=\zeta\left(-\beta_{i}, \alpha_{i}, \chi_{i}\right)$. Hence, our assumptions are $\zeta_{i} \times \zeta_{j}, \zeta_{i} \times \widetilde{\zeta}_{j}(i \neq j)$, 
$\zeta_{i} \rtimes \sigma_{\text {neg }}, i, j=1, \ldots, k$, are irreducible. Now, we see (using Corollary 4.1)

$$
\zeta_{1} \times \cdots \times \zeta_{k} \rtimes \sigma_{n e g} \simeq \widetilde{\zeta}_{1} \times \cdots \times \widetilde{\zeta}_{k} \rtimes \sigma_{n e g} .
$$

Let us write $\pi$ for the unique irreducible subrepresentation $\zeta_{1} \times \cdots \times \zeta_{k} \rtimes \sigma_{n e g}$. We want to prove $\pi \simeq \widetilde{\pi}$. This is clear for orthogonal groups using the work of Waldspurger (cf. MVW]). Also by the work of Waldspurger (cf. MVW]), if $G_{n}=S p(n, F)$, then there exists an element $\eta \in G S p(n, F)$ of similitude -1 , such that the representation $\pi^{\eta}(g):=\pi\left(\eta g \eta^{-1}\right)$ is isomorphic to the contragredient of $\pi$. Now, if we choose $\eta$ appropriately, then we have

$$
\pi^{\eta} \hookrightarrow \zeta_{1} \times \cdots \times \zeta_{k} \rtimes \sigma_{n e g}^{\eta} \cong \zeta_{1} \times \cdots \times \zeta_{k} \rtimes \sigma_{n e g} .
$$

Hence $\pi^{\eta} \simeq \pi$.

Next, since $\widetilde{\pi} \simeq \pi, \widetilde{\sigma}_{n e g} \simeq \sigma_{n e g}$, and

$$
\left(\zeta_{1} \times \cdots \times \zeta_{k} \rtimes \sigma_{n e g}\right)^{\sim} \simeq \widetilde{\zeta}_{1} \times \cdots \times \widetilde{\zeta}_{k} \rtimes \sigma_{n e g},
$$

we see that $\pi$ appears as a subrepresentation and as a quotient of $\zeta_{1} \times \cdots \times \zeta_{k} \rtimes \sigma_{n e g}$, but since it appears with multiplicity one and is a unique irreducible subrepresentation there, it must be

$$
\pi \simeq \zeta_{1} \times \cdots \times \zeta_{k} \rtimes \sigma_{n e g} .
$$

In Section 5 we will prove the following fundamental result (see Corollary 4.2 and Lemma 5.6).

Theorem 4.2. Assume that $\sigma \in \operatorname{Irr} G_{n}$ is a negative unramified representation. Let $\alpha, \beta \in \mathbb{R}, \alpha+\beta \in \mathbb{Z}_{\geq 0}, \alpha>\beta$, and let $\chi$ be the unramified character of $F^{\times}$. Then $\zeta(-\beta, \alpha, \chi) \rtimes \sigma$ reduces if and only if a unique irreducible unramified subquotient of $\zeta(-\beta, \alpha, \chi) \rtimes \sigma$ is not its (unique) irreducible subrepresentation.

Assuming this we complete the classification of unramified representations in terms of negative representations.

Theorem 4.3. Assume that $\sigma \in \operatorname{Irr} G_{n}$ is an unramified representation. Then there exists a unique (up to permutation and taking inverses) sequence of unramified characters $\chi_{1} \mathbf{1}_{G L\left(n_{1}, F\right)}, \ldots, \chi_{k} \mathbf{1}_{G L\left(n_{k}, F\right)}$ and a negative unramified representation $\sigma_{\text {neg }}$ such that $e\left(\chi_{i}\right) \neq 0, i=1, \ldots, k$, and

$$
\sigma \simeq \chi_{1} \mathbf{1}_{G L\left(n_{1}, F\right)} \times \cdots \times \chi_{k} \mathbf{1}_{G L\left(n_{k}, F\right)} \rtimes \sigma_{n e g} .
$$

Proof. According to Lemma 4.7, there is a unique up to permutation sequence of unramified characters $\chi_{1} \mathbf{1}_{G L\left(n_{1}, F\right)}, \ldots, \chi_{k} \mathbf{1}_{G L\left(n_{k}, F\right)}$ and a negative unramified representation $\sigma_{\text {neg }}$ such that $e\left(\chi_{i}\right)>0, i=1, \ldots, k$,

$$
\sigma \hookrightarrow \chi_{1} \mathbf{1}_{G L\left(n_{1}, F\right)} \times \cdots \times \chi_{k} \mathbf{1}_{G L\left(n_{k}, F\right)} \rtimes \sigma_{n e g} .
$$

Furthermore, the unramified representation $\sigma$ is a unique subrepresentation there, and

$$
\chi_{1} \mathbf{1}_{G L\left(n_{1}, F\right)} \times \cdots \times \chi_{k} \mathbf{1}_{G L\left(n_{k}, F\right)}
$$

is irreducible. 
Next, we show that $\chi_{i} \mathbf{1}_{G L\left(n_{i}, F\right)} \rtimes \sigma_{n e g}$ is irreducible for all $i$. If not, then according to Lemma 4.8, $\chi_{i} \mathbf{1}_{G L\left(n_{i}, F\right)} \rtimes \sigma_{n e g}$ has a unique irreducible subrepresentation, say $\pi$, and, according to Theorem $4.2, \pi$ is not unramified. Now, since $\chi_{1} \mathbf{1}_{G L\left(n_{1}, F\right)} \times \cdots \times \chi_{k} \mathbf{1}_{G L\left(n_{k}, F\right)}$ is irreducible we have

$$
\begin{array}{r}
\chi_{1} \mathbf{1}_{G L\left(n_{1}, F\right)} \times \cdots \times \chi_{i-1} \mathbf{1}_{G L\left(n_{i-1}, F\right)} \times \chi_{i+1} \mathbf{1}_{G L\left(n_{i+1}, F\right)} \times \cdots \times \chi_{k} \mathbf{1}_{G L\left(n_{k}, F\right)} \rtimes \pi \\
\hookrightarrow \chi_{1} \mathbf{1}_{G L\left(n_{1}, F\right)} \times \cdots \times \chi_{i-1} \mathbf{1}_{G L\left(n_{i-1}, F\right)} \times \chi_{i+1} \mathbf{1}_{G L\left(n_{i+1}, F\right)} \times \cdots \times \chi_{k} \mathbf{1}_{G L\left(n_{k}, F\right)} \\
\times \chi_{i} \mathbf{1}_{G L\left(n_{i}, F\right)} \rtimes \sigma_{n e g} \simeq \chi_{1} \mathbf{1}_{G L\left(n_{1}, F\right)} \times \cdots \times \chi_{k} \mathbf{1}_{G L\left(n_{k}, F\right)} \rtimes \sigma_{n e g} .
\end{array}
$$

Hence

$$
\begin{aligned}
\sigma \hookrightarrow \chi_{1} \mathbf{1}_{G L\left(n_{1}, F\right)} \times \cdots \times \chi_{i-1} \mathbf{1}_{G L\left(n_{i-1}, F\right)} & \\
& \times \chi_{i+1} \mathbf{1}_{G L\left(n_{i+1}, F\right)} \times \cdots \times \chi_{k} \mathbf{1}_{G L\left(n_{k}, F\right)} \rtimes \pi .
\end{aligned}
$$

This contradicts Lemma 1.1(ii) since $\pi$ is not unramified. Thus, $\chi_{i} \mathbf{1}_{G L\left(n_{i}, F\right)} \rtimes \sigma_{\text {neg }}$ is irreducible for all $i$. Next, we similarly show that $\chi_{i} \mathbf{1}_{G L\left(n_{i}, F\right)} \times \chi_{j}^{-1} \mathbf{1}_{G L\left(n_{j}, F\right)}$ is irreducible. Observing that Lemma 2.1 implies a unique irreducible subrepresentation of $\chi_{i} \mathbf{1}_{G L\left(n_{i}, F\right)} \times \chi_{j}^{-1} \mathbf{1}_{G L\left(n_{j}, F\right)}$ is not unramified. We leave details to the reader. Now, Lemma 4.8 implies that $\chi_{1} \mathbf{1}_{G L\left(n_{1}, F\right)} \times \cdots \times \chi_{k} \mathbf{1}_{G L\left(n_{k}, F\right)} \rtimes \sigma_{\text {neg }}$ is irreducible. Finally, the remainder of the theorem follows combining Lemma 4.7 and Lemma 4.8.

It remains to discuss and to classify negative representations. The next theorem is the first step.

Theorem 4.4. Assume that $\sigma \in \operatorname{Irr} G_{n}$ is a negative unramified representation. Then there exist a strongly negative unramified representation $\sigma_{s n}$ and a sequence of unitary characters $\chi_{1} \mathbf{1}_{G L\left(n_{1}, F\right)}, \ldots, \chi_{k} \mathbf{1}_{G L\left(n_{k}, F\right)}$ such that

$$
\sigma \hookrightarrow \chi_{1} \mathbf{1}_{G L\left(n_{1}, F\right)} \times \cdots \times \chi_{k} \mathbf{1}_{G L\left(n_{k}, F\right)} \rtimes \sigma_{s n} .
$$

Proof. If $\sigma$ is strongly negative, then $\sigma \simeq \sigma_{s n}$ and we are done. Thus assume that $\sigma$ is not strongly negative. Then, we can find unramified characters $\chi_{1}, \ldots, \chi_{n}$ such that

$$
\operatorname{Jacq}_{(1, \ldots, 1 ; 0)}(\sigma) \geq \chi_{1} \otimes \cdots \otimes \chi_{n}
$$

and $1 \leq k \leq n$ such that

$$
e\left(\chi_{1}\right)+\cdots+e\left(\chi_{k}\right)=0 .
$$

In fact, we have

which is equivalent to

$$
\operatorname{Jacq}_{(1, \ldots, 1 ; n)}(\sigma) \rightarrow \chi_{1} \otimes \cdots \otimes \chi_{n},
$$

$$
\sigma \hookrightarrow \chi_{1} \times \cdots \times \chi_{n} \rtimes \mathbf{1} .
$$

Now, we partition the set of indices $\{1, \ldots, k\}$. We say that $i$ and $j$ are equivalent if $\chi_{i}^{u}=\chi_{j}^{u}$. This is clearly a relation of equivalence on $\{1, \ldots, k\}$. Thus, for each of its partitions $\mathcal{J}$ we must have

$$
\sum_{i \in \mathcal{J}} e\left(\chi_{i}\right) \leq 0 .
$$

Otherwise, if for some $\mathcal{J}$ we have $\sum_{i \in \mathcal{J}} e\left(\chi_{i}\right)>0$, then let us write $\mathcal{J}=\left\{i_{1}, \ldots, i_{l}\right\}$, $i_{1}<i_{2}<\cdots<i_{l}$. Then since [Ze] or Lemma 2.1 here imply that

$$
\chi_{i} \times \chi_{j} \cong \chi_{j} \times \chi_{i}, \quad i \in \mathcal{J}, j \notin \mathcal{J}, 1 \leq j \leq k,
$$


we have

$$
\sigma \hookrightarrow \chi_{i_{1}} \times \cdots \times \chi_{i_{l}} \times \text { (other characters in the same order) } \rtimes \mathbf{1},
$$

and this contradicts negativity.

Now, summing over all partitions, using (4.26) $e\left(\chi_{1}\right)+\cdots+e\left(\chi_{k}\right)=0$, we conclude that for any partition $\mathcal{J}$ we must have

$$
\sum_{i \in \mathcal{J}} e\left(\chi_{i}\right)=0 .
$$

Now, we fix a partition $\mathcal{J}$. Let us write $\mathcal{J}=\left\{i_{1}, \ldots, i_{l}\right\}, i_{1}<i_{2}<\cdots<i_{l}$. As before, (4.27) holds, and (4.27) can be rewritten using Lemma 4.3, as follows:

$$
\sigma \hookrightarrow \pi \times \text { (other characters in the same order }) \rtimes \mathbf{1}
$$

where $\pi$ is the unique unramified irreducible subquotient of $\chi_{i_{1}} \times \cdots \times \chi_{i_{l}}$. Now, we apply Lemma 2.1 and Theorem 2.1 to write

$$
\pi \simeq \zeta\left(-\beta_{1}^{\prime}, \alpha_{1}^{\prime}, \chi_{1}^{\prime}\right) \times \cdots \times \zeta\left(-\beta_{k^{\prime}}^{\prime}, \alpha_{k^{\prime}}^{\prime}, \chi_{k^{\prime}}^{\prime}\right),
$$

where $\alpha_{i}^{\prime}, \beta_{i}^{\prime} \in \mathbb{R}$ and $\chi_{i}^{\prime}$ is unitary unramified character, $i=1, \ldots, k$. We can argue as in the first part of the proof to see that $\alpha_{i}^{\prime}=\beta_{i}^{\prime}$, for all $i$. Since $\chi_{1}^{\prime} \mathbf{1}_{G L\left(2 \alpha_{i}^{\prime}+1, F\right)}=$ $\zeta\left(-\alpha_{1}^{\prime}, \alpha_{1}^{\prime}, \chi_{1}^{\prime}\right)$, we see that (4.29) implies

$$
\sigma \hookrightarrow \chi_{1}^{\prime} \mathbf{1}_{G L\left(2 \alpha_{i}^{\prime}+1, F\right)} \times \cdots
$$

Now, we apply the induction to get the result.

Corollary 4.2. Assume that $\sigma \in \operatorname{Irr} G_{n}$ is a negative unramified representation given by the data from Theorem 4.4. Assume that $\alpha, \beta \in \mathbb{R}, \alpha+\beta \in \mathbb{Z}_{\geq 0}, \alpha>\beta$, and $\chi$ is a unitary character of $F^{\times}$. Then we have that $\zeta(-\beta, \alpha, \chi) \rtimes \sigma$ reduces if and only if $\zeta(-\beta, \alpha, \chi) \rtimes \chi_{i}^{ \pm 1} \mathbf{1}_{G L\left(n_{i}, F\right)}$ reduces for some index $i$ and for some choice of sign \pm , or $\zeta(-\beta, \alpha, \chi) \rtimes \sigma_{\text {sn }}$ reduces. Moreover, if $\zeta(-\beta, \alpha, \chi) \rtimes \sigma_{\text {sn }}$ reduces and its (unique) irreducible subrepresentation is not unramified, then $\zeta(-\beta, \alpha, \chi) \rtimes \sigma$ reduces and its (unique) irreducible subrepresentation is not unramified.

Proof. We leave it to the reader to make necessary modifications in the proof of Lemma 4.8 and Theorem 4.3 to prove this fact.

Corollary 4.2 reduces the proof of Theorem 4.2 from the case of negative unramified representations to the case of strongly negative unramified representations. Lemma 5.6 completes the proof of Theorem 4.2.

\section{Strongly NeGAtive UnRAmified Representations}

In this section we classify the strongly negative unramified representations. We are motivated by the approach to the classification of discrete series due to Moeglin and Tadić (cf. [T3], Moe, MT]).

First, we define the notion of the Jordan block, following Moeglin (cf. [Moe]), as the pair $(m, \chi)$, where $m \in \mathbb{Z}_{>0}$ and $\chi \in\left\{\mathbf{1}, \chi_{0}\right\}$ (see Theorem 3.3 for the notation) such that $m$ is even if and only if we deal with odd-orthogonal groups (and odd if and only if we deal with symplectic or even-orthogonal groups). We say that $m$ is a size of the Jordan block. 
Now, we fix $n \in \mathbb{Z}_{>0}$ and denote by $\operatorname{Irr}_{s n}\left(G_{n}\right)$ the set of equivalence classes of strongly negative irreducible unramified representations of $G_{n}$. We write $\operatorname{Jord}(n)$ for the collection of all sets of Jordan blocks Jord $:=\{(m, \chi)\}$ such that

$$
\sum_{(m, \chi) \in \text { Jord }} m=\left\{\begin{array}{l}
2 n \quad \text { if } G_{n}=S O(2 n+1, F), O(2 n, F), \\
2 n+1 \quad \text { if } G_{n}=S p(n, F) .
\end{array}\right.
$$

Let $\chi \in\left\{\mathbf{1}, \chi_{0}\right\}$; we put $\operatorname{Jord}(\chi)$ for the set of all $m$ such that $(m, \chi) \in$ Jord. We also require that \# $\operatorname{Jord}(\chi)$ (resp., \# $\operatorname{Jord}\left(\chi_{0}\right)$ ) is even for $G_{n}=O(2 n, F)$ (resp., $G_{n}=S p(n, F)$ ). Then we write $\operatorname{Jord}(\chi)$ according to the character $\chi$ (the case $l=0$ or $k=0$ is not excluded):

$$
\left\{\begin{array}{l}
\chi=1: \quad 2 m_{1}+1<2 m_{2}+1<\cdots<2 m_{l}+1, \\
\chi=\chi_{0}: \quad 2 n_{1}+1<2 n_{2}+1<\cdots<2 n_{k}+1
\end{array}\right.
$$

where $m_{i}, n_{j} \in \mathbb{Z}_{\geq 0}\left(G_{n}=O(2 n, F), S p(n, F)\right)$ or $m_{i}, n_{j} \in \frac{1}{2}+\mathbb{Z}_{\geq 0}\left(G_{n}=\right.$ $S O(2 n+1, F))$, and

$$
\sum_{i}\left(2 m_{i}+1\right)+\sum_{j}\left(2 n_{j}+1\right)=\left\{\begin{array}{l}
2 n \text { if } G_{n}=S O(2 n+1, F), O(2 n, F), \\
2 n+1 \text { if } G_{n}=S p(2 n, F) .
\end{array}\right.
$$

In any case, we associate to Jord an unramified irreducible representation $\sigma($ Jord $)$ uniquely requiring the following:

$$
\begin{aligned}
\zeta\left(-m_{l-1}, m_{l}, \mathbf{1}\right) \times \zeta\left(-m_{l-3}, m_{l-2}, \mathbf{1}\right) \times \cdots \times \zeta\left(-n_{k-1}, n_{k}, \chi_{0}\right) \\
\times \zeta\left(-n_{k-3}, n_{k-2}, \chi_{0}\right) \times \cdots \rtimes \sigma_{0}(\text { Jord }) \geq \sigma(\text { Jord }),
\end{aligned}
$$

where $\sigma_{0}($ Jord $)$ is a unique irreducible unramified representation such that

$$
\left\{\begin{array}{l}
\zeta\left(1, m_{1}, \mathbf{1}\right) \rtimes \mathbf{1} \text { if } l \in 2 \mathbb{Z}+1, G_{n}=S p(n, F), \\
\zeta\left(1 / 2, m_{1}, \mathbf{1}\right) \times \zeta\left(1 / 2, n_{1}, \chi_{0}\right) \rtimes \mathbf{1} \text { if } k, l \in 2 \mathbb{Z}+1, G_{n}=S O(2 n+1, F), \\
\zeta\left(1 / 2, m_{1}, \mathbf{1}\right) \rtimes \mathbf{1} \text { if } k \in 2 \mathbb{Z}+1, l \in 2 \mathbb{Z}, G_{n}=S O(2 n+1, F), \\
\zeta\left(1 / 2, n_{1}, \chi_{0}\right) \rtimes \mathbf{1} \text { if } k \in 2 \mathbb{Z}, l \in 2 \mathbb{Z}+1, G_{n}=S O(2 n+1, F), \\
\mathbf{1} \in \operatorname{Irr} G_{0} \text { if } k, l \in 2 \mathbb{Z} .
\end{array}\right.
$$

The main result of this section is the next theorem.

Theorem 5.1. Let $n \in \mathbb{Z}_{>0}$. The correspondence $\operatorname{Jord}(n) \rightarrow \operatorname{Irr}_{s n}\left(G_{n}\right)$ given by Jord $\rightsquigarrow \sigma$ (Jord) is a well-defined bijection.

Remark 5.1. If $G_{n}=S p(n, F)$, then $G_{0}$ stands for the trivial group. In that case its only representation $\mathbf{1}$ is also considered to be a strongly negative unramified representation. We put $\operatorname{Jord}(\mathbf{1})=\{(\mathbf{1}, \mathbf{1})\}$. Now, the theorem also holds for $n=0$ in the symplectic case.

The proof of Theorem 5.1 will be done in several steps. We prove that the map Jord $\rightsquigarrow \sigma$ (Jord) is well defined in Lemma 5.3. Then the explicit construction of the map Jord $\rightsquigarrow \sigma$ (Jord) shows its injectivity. Finally, we prove the surjectivity in Lemma 5.7.

We start with the next lemma computing Jacquet modules. 
Lemma 5.1. Let $\chi \in\left\{1, \chi_{0}\right\}$. Assume that $\beta \in\{1 / 2,1\}$ is such that $\nu^{\beta} \chi \rtimes 1$ reduces. Let $\alpha \in \mathbb{R}$ such that $\alpha-\beta \in \mathbb{Z}_{\geq 0}$. We define $\sigma_{\alpha}$ as the unique unramified irreducible subquotient of $\zeta(\beta, \alpha, \chi) \rtimes \mathbf{1}$. Then $\sigma_{\alpha}$ is strongly negative, and we have the following:

$$
\mu^{*}\left(\sigma_{\alpha}\right)=\sum_{i=0}^{\alpha-\beta+1} \zeta(-\alpha,-\beta-i, \chi) \otimes \sigma_{i+\beta-1} .
$$

Here we put $\sigma_{\beta-1}=\mathbf{1}$.

Proof. We prove the claim using induction on $\alpha$. First, $\alpha=\beta$. Then the formula in (5.4) reduces to $\mu^{*}\left(\sigma_{\beta}\right)=\nu^{-\beta} \chi \otimes \mathbf{1}+1 \otimes \sigma_{\beta}$, which is well known.

Now, we assume that the formula holds for all $\beta \leq \alpha^{\prime}<\alpha$, and we prove it for $\alpha$. We first show that $\sigma_{\alpha}$ is strongly negative. If not, then by Lemma 4.7 and Theorem 4.4, we discuss two separate cases: $\sigma_{\alpha}$ is negative but not strongly negative, and $\sigma_{\alpha}$ is not negative. We start with the first case. Using Theorem 4.4 , we can find $2 m \in \mathbb{Z}_{\geq 0}$, an unramified unitary character $\chi^{\prime}$, and an irreducible negative representation $\bar{\sigma}^{\prime}$ such that

$$
\sigma \hookrightarrow \zeta\left(-m, m, \chi^{\prime}\right) \rtimes \sigma^{\prime} .
$$

Hence, by Frobenius reciprocity, $\mu^{*}\left(\sigma_{\alpha}\right) \geq \zeta\left(-m, m, \chi^{\prime}\right) \otimes \sigma^{\prime}$. Since, $\sigma_{\alpha} \leq \zeta(\beta, \alpha, \chi)$ $\rtimes \mathbf{1}$, we see that

$$
\zeta\left(-m, m, \chi^{\prime}\right) \otimes \sigma^{\prime} \leq \mu^{*}(\zeta(\beta, \alpha, \chi) \rtimes \mathbf{1}) .
$$

Now, using Theorem 3.1, we can find indices $0 \leq j \leq i \leq \alpha-\beta+1$ such that

$\zeta\left(-m, m, \chi^{\prime}\right) \otimes \sigma^{\prime} \leq \zeta(-\alpha,-\beta-i, \chi) \times \zeta(\beta, j+\beta-1, \chi) \otimes \zeta(j+\beta, i+\beta-1, \chi) \rtimes \mathbf{1}$.

Hence, we see that $\chi^{\prime}=\chi$. Also, if $i<\alpha-\beta+1$, we see that $m=\alpha$ and $m=j+\beta-1$. Thus, $i<\alpha-\beta+1=j$. This is a contradiction. Thus, $i=\alpha-\beta+1$. Hence $\beta=m=0$ and $j=1$. Hence $\sigma^{\prime} \leq \zeta(\beta+1, \alpha, \chi) \rtimes \mathbf{1}$. Since Theorem 3.2 implies that $\zeta(\beta+1, \alpha, \chi) \rtimes \mathbf{1}$ is irreducible, we see that $\sigma^{\prime} \simeq \zeta(\beta+1, \alpha, \chi) \rtimes \mathbf{1}$, contradicting the negativity of $\sigma^{\prime}$. Thus, we consider the case $\sigma_{\alpha}$ is not negative. Now, we realize $\sigma_{\alpha}$ as an irreducible subrepresentation of (4.13). Thus, as in the proof we just completed, we can find indices $0 \leq j \leq i \leq \alpha-\beta+1$ such that

$$
\begin{aligned}
& \zeta\left(-\beta_{1}, \alpha_{1}, \chi_{1}\right) \times \cdots \times \zeta\left(-\beta_{k}, \alpha_{k}, \chi_{k}\right) \otimes \sigma_{n e g} \\
& \leq \zeta(-\alpha,-\beta-i, \chi) \times \zeta(\beta, j+\beta-1, \chi) \otimes \zeta(j+\beta, i+\beta-1, \chi) \rtimes \mathbf{1} .
\end{aligned}
$$

Since $\alpha_{i}-\beta_{i}>0$ and $-\alpha-\beta-i \leq 0$, we see that $i=\alpha-\beta+1$ and $j>0$. Hence $k=1$ and, as before, $\sigma_{n e g} \simeq \zeta(\beta+j, \alpha, \chi) \rtimes \mathbf{1}$. This contradicts the negativity unless $j=\alpha-\beta+1$. Hence $\zeta\left(-\beta_{1}, \alpha_{1}, \chi_{1}\right)=\zeta(\beta, \alpha, \chi)$ and $\sigma_{\text {neg }}=\mathbf{1}$. Thus,

$$
\sigma_{\alpha} \hookrightarrow \zeta(\beta, \alpha, \chi) \rtimes \mathbf{1} .
$$

Hence

$$
\begin{aligned}
\sigma_{\alpha} \hookrightarrow \zeta(\beta, \alpha, \chi) \rtimes \mathbf{1} & \hookrightarrow \zeta(\beta, \alpha-1, \chi) \times \nu^{\alpha} \chi \rtimes \mathbf{1} \\
& \simeq \zeta(\beta, \alpha-1, \chi) \times \nu^{-\alpha} \chi \rtimes \mathbf{1} \simeq \nu^{-\alpha} \chi \times \zeta(\beta, \alpha-1, \chi) \rtimes \mathbf{1} .
\end{aligned}
$$

Now, one can prove directly that $\nu^{-\alpha} \chi \times \zeta(\beta, \alpha-1, \chi) \rtimes \mathbf{1}$ has a unique irreducible subrepresentation using Theorem 3.1 and using an idea similar to that used in the proof of Lemma 4.7. Hence for the unique irreducible representation $\sigma^{\prime}$ of $\zeta(\beta, \alpha-1, \chi) \rtimes \mathbf{1}$, we must have $\sigma_{\alpha} \hookrightarrow \nu^{-\alpha} \chi \rtimes \sigma^{\prime}$. This contradicts Lemma 1.1(ii), 
since $\sigma^{\prime}$ is not unramified. (Since by Frobenius reciprocity, $\mu^{*}\left(\sigma^{\prime}\right) \geq \zeta(\beta, \alpha-1, \chi) \otimes$ $\mathbf{1}$, and thus $\sigma^{\prime} \not \sigma_{\alpha-1}$.) Thus, we have proved that $\sigma_{\alpha}$ is strongly negative.

Now, we prove (5.4). First, considering uniqueness of unramified irreducible subquotients we see that

$$
\sigma_{\alpha} \leq \nu^{\alpha} \chi \rtimes \sigma_{\alpha-1} .
$$

Hence

$$
\begin{aligned}
& \mu^{*}\left(\sigma_{\alpha}\right) \leq \mu^{*}\left(\nu^{\alpha} \chi \rtimes \sigma_{\alpha-1}\right) \\
& \quad=\left(\nu^{\alpha} \chi \otimes \mathbf{1}+\nu^{-\alpha} \chi \otimes 1+\mathbf{1} \otimes \nu^{\alpha} \chi\right) \rtimes \sum_{i=0}^{\alpha-\beta} \zeta(-\alpha+1,-\beta-i, \chi) \otimes \sigma_{i+\beta-1} .
\end{aligned}
$$

Since $\sigma_{\alpha}$ is strongly negative, we must have

$$
\mu^{*}\left(\sigma_{\alpha}\right) \leq\left(\nu^{-\alpha} \chi \otimes 1+\mathbf{1} \otimes \nu^{\alpha} \chi\right) \rtimes \sum_{i=0}^{\alpha-\beta} \zeta(-\alpha+1,-\beta-i, \chi) \otimes \sigma_{i+\beta-1} .
$$

We show that $\mathbf{1} \otimes \sigma_{\alpha}$ is the only term in $\mu^{*}\left(\sigma_{\alpha}\right)$ less than

$$
\sum_{i=0}^{\alpha-\beta} \zeta(-\alpha+1,-\beta-i, \chi) \otimes \nu^{\alpha} \chi \rtimes \sigma_{i+\beta-1} .
$$

This case happens with $i=\alpha-\beta$. If $i<\alpha-\beta$, then

$$
\mu^{*}\left(\sigma_{\alpha}\right) \geq \zeta(-\alpha+1,-\beta-i, \chi) \otimes \nu^{\alpha} \chi \rtimes \sigma_{i+\beta-1}
$$

and $\nu^{\alpha} \chi \rtimes \sigma_{i+\beta-1}$ is irreducible since we have the following isomorphisms:

$$
\begin{aligned}
\nu^{\alpha} \chi \rtimes \sigma_{i+\beta-1} \hookrightarrow \nu^{\alpha} \chi \times \tilde{\zeta}(\beta, i+\beta-1, \chi) \rtimes \mathbf{1} \simeq \tilde{\zeta}(\beta, i+\beta-1, \chi) \times \nu^{\alpha} \chi \rtimes \mathbf{1} \\
\simeq \tilde{\zeta}(\beta, i+\beta-1, \chi) \times \nu^{-\alpha} \chi \rtimes \mathbf{1} \simeq \nu^{-\alpha} \chi \times \tilde{\zeta}(\beta, i+\beta-1, \chi) \rtimes \mathbf{1} .
\end{aligned}
$$

Since also

$$
\nu^{-\alpha} \chi \rtimes \sigma_{i+\beta-1} \hookrightarrow \nu^{-\alpha} \chi \times \tilde{\zeta}(\beta, i+\beta-1, \chi) \rtimes \mathbf{1},
$$

we can argue as in the proof of Lemma 4.8 to show the irreducibility. We leave the details to the reader.

Now, Lemma 4.1 applied to $\mu^{*}\left(\sigma_{\alpha}\right) \geq \zeta(-\alpha+1,-\beta-i, \chi) \otimes \nu^{\alpha} \chi \rtimes \sigma_{i+\beta-1}$ shows

$$
\sigma_{\alpha} \hookrightarrow \zeta(-\alpha+1,-\beta-i, \chi) \times \nu^{\alpha} \chi \rtimes \sigma_{i+\beta-1} .
$$

Hence

$\sigma_{\alpha} \hookrightarrow \zeta(-\alpha+1,-\beta-i, \chi) \times \nu^{\alpha} \chi \rtimes \sigma_{i+\beta-1} \simeq \nu^{\alpha} \chi \times \zeta(-\alpha+1,-\beta-i, \chi) \rtimes \sigma_{i+\beta-1}$.

Thus $\mu^{*}\left(\sigma_{\alpha}\right) \geq \nu^{\alpha} \chi \otimes \cdots$, contradicting the strong negativity of $\sigma_{\alpha}$. Thus we get the following inequality:

$$
\mu^{*}\left(\sigma_{\alpha}\right) \leq 1 \otimes \sigma_{\alpha}+\sum_{i=0}^{\alpha-\beta} \nu^{-\alpha} \chi \times \zeta(-\alpha+1,-\beta-i, \chi) \otimes \sigma_{i+\beta-1} .
$$

Note that for any $i, 0 \leq i \leq \alpha-\beta$, we write

$$
\zeta(-\alpha,-\beta-i, \chi) \hookrightarrow \nu^{-\alpha} \chi \times \zeta(-\alpha+1,-\beta-i, \chi) \rightarrow \zeta,
$$

where $\zeta$ is irreducible and not spherical. Moreover, by [Ze], we have

$$
\zeta \hookrightarrow \zeta(-\alpha+1,-\beta-i, \chi) \times \nu^{-\alpha} \chi .
$$


Thus, if $\mu^{*}\left(\sigma_{\alpha}\right) \geq \zeta \otimes \sigma_{i+\beta-1}$, we see that

$$
\sigma_{\alpha} \hookrightarrow \nu^{-\alpha^{+} 1} \chi \times \cdots \times \nu^{-\beta-i} \times \nu^{-\alpha} \chi \rtimes \sigma_{i+\beta-1}
$$

by Lemma 4.3. Since we proved that $\nu^{\alpha} \chi \rtimes \sigma_{i+\beta-1}$ is irreducible, we have

$$
\begin{aligned}
\sigma_{\alpha} & \hookrightarrow \nu^{-\alpha+1} \chi \times \cdots \times \nu^{-\beta-i} \chi \times \nu^{-\alpha} \chi \rtimes \sigma_{i+\beta-1} \\
& \simeq \nu^{-\alpha+1} \chi \times \cdots \times \nu^{-\beta-i} \chi \times \nu^{\alpha} \chi \rtimes \sigma_{i+\beta-1},
\end{aligned}
$$

and since $\nu^{j} \chi \times \nu^{\alpha} \chi \simeq \nu^{\alpha} \chi \times \nu^{j} \chi,-\alpha+1 \leq j \leq-\beta-i$, we obtain $\mu^{*}\left(\sigma_{\alpha}\right) \geq$ $\nu^{\alpha} \chi \otimes \cdots$, contradicting the strong negativity of $\sigma_{\alpha}$. This proves (5.4) and completes the proof of the lemma.

The next lemma shows the strong negativity of all unramified representations in (5.3). It also computes their Jacquet modules.

Lemma 5.2. Assume that $\beta, \beta^{\prime} \in\{1 / 2,1\}$ such that $\nu^{\beta} \rtimes 1$ and $\nu^{\beta^{\prime}} \chi_{0} \rtimes 1$ reduce. Let $\alpha, \alpha^{\prime} \in \mathbb{R}$ such that $\alpha-\beta \in \mathbb{Z}_{\geq 0}$ and $\alpha^{\prime}-\beta^{\prime} \in \mathbb{Z}_{\geq 0}$. We define $\sigma_{\alpha, \alpha^{\prime}}$ as the unique unramified irreducible subquotient of $\zeta(\beta, \alpha, \mathbf{1}) \times \zeta\left(\beta^{\prime}, \alpha^{\prime}, \chi_{0}\right) \rtimes \mathbf{1}$, and the unique unramified irreducible subquotients of $\zeta(\beta, \alpha, \mathbf{1}) \rtimes \mathbf{1}$ and $\zeta\left(\beta^{\prime}, \alpha^{\prime}, \chi_{0}\right) \rtimes \mathbf{1}$ we denote by $\sigma_{\alpha}$ and $\sigma_{\alpha}^{\prime}$, respectively. Obviously, we have the following:

$$
\left\{\begin{array}{l}
\sigma_{\alpha, \alpha^{\prime}} \leq \zeta(\beta, \alpha, \mathbf{1}) \rtimes \sigma_{\alpha^{\prime}}^{\prime} \\
\sigma_{\alpha, \alpha^{\prime}} \leq \zeta\left(\beta^{\prime}, \alpha^{\prime}, \chi_{0}\right) \rtimes \sigma_{\alpha} .
\end{array}\right.
$$

Then $\sigma_{\alpha, \alpha^{\prime}}$ is strongly negative and we have the following:

$\mu^{*}\left(\sigma_{\alpha, \alpha^{\prime}}\right)=\sum_{i=0}^{\alpha-\beta+1} \sum_{i^{\prime}=0}^{\alpha^{\prime}-\beta^{\prime}+1} \zeta(-\alpha,-\beta-i, \mathbf{1}) \times \zeta\left(-\alpha^{\prime},-\beta^{\prime}-i^{\prime}, \chi_{0}\right) \otimes \sigma_{i+\beta-1, i^{\prime}+\beta^{\prime}-1}$.

Proof. We prove by induction on $\alpha+\alpha^{\prime}$ that if an irreducible representation $\sigma$ satisifies

$$
\left\{\begin{array}{l}
\sigma \leq \zeta(\beta, \alpha, \mathbf{1}) \rtimes \sigma_{\alpha^{\prime}}^{\prime} \\
\sigma \leq \zeta\left(\beta^{\prime}, \alpha^{\prime}, \chi_{0}\right) \rtimes \sigma_{\alpha}
\end{array}\right.
$$

then $\sigma \simeq \sigma_{\alpha, \alpha^{\prime}}$. The formula for Jacquet modules in (5.6) will be the consequence. The base of induction is the case $\alpha=\beta-1$ or $\alpha^{\prime}=\beta^{\prime}-1$; then the conclusion trivially holds. In general, the first inequality in (5.6), Lemma 5.1, and Theorem 3.1 imply

$$
\begin{aligned}
\mu^{*}(\sigma) \leq \sum_{i=0}^{\alpha-\beta+1} \sum_{j=0}^{i} \sum_{i^{\prime}=0}^{\alpha^{\prime}-\beta^{\prime}+1} \zeta(-\alpha,-\beta-i, \mathbf{1}) \times \zeta(\beta, j+\beta-1, \mathbf{1}) \\
\quad \times \zeta\left(-\alpha^{\prime},-\beta^{\prime}-i^{\prime}, \chi_{0}\right) \otimes \zeta(j+\beta, i+\beta-1, \mathbf{1}) \rtimes \sigma_{i^{\prime}+\beta^{\prime}-1}^{\prime}
\end{aligned}
$$

and

$$
\begin{aligned}
\mu^{*}(\sigma) \leq \sum_{i=0}^{\alpha-\beta+1} & \sum_{i^{\prime}=0}^{\alpha^{\prime}-\beta^{\prime}+1} \sum_{j^{\prime}=0}^{i^{\prime}} \zeta(-\alpha,-\beta-i, \mathbf{1}) \times \zeta\left(-\alpha^{\prime},-\beta^{\prime}-i^{\prime}, \chi_{0}\right) \\
& \times \zeta\left(\beta^{\prime}, j^{\prime}+\beta^{\prime}-1, \chi_{0}\right) \otimes \zeta\left(j^{\prime}+\beta^{\prime}, i^{\prime}+\beta^{\prime}-1, \chi_{0}\right) \rtimes \sigma_{i+\beta-1} .
\end{aligned}
$$


A comparison of those formulas shows $j=j^{\prime}=0$. Thus, $\mu^{*}\left(\sigma_{\alpha, \alpha^{\prime}}\right)$ is less than or equal to both

$$
\left\{\begin{aligned}
\sum_{i=0}^{\alpha-\beta+1} \sum_{i^{\prime}=0}^{\alpha^{\prime}-\beta^{\prime}+1} \zeta(-\alpha,-\beta-i, \mathbf{1}) & \times \zeta\left(-\alpha^{\prime},-\beta^{\prime}-i^{\prime}, \chi_{0}\right) \\
& \otimes \zeta(\beta, i+\beta-1, \mathbf{1}) \rtimes \sigma_{i^{\prime}+\beta^{\prime}-1}^{\prime}, \\
\sum_{i=0}^{\alpha-\beta+1} \sum_{i^{\prime}=0}^{\alpha^{\prime}-\beta^{\prime}+1} \zeta(-\alpha,-\beta-i, \mathbf{1}) & \times \zeta\left(-\alpha^{\prime},-\beta^{\prime}-i^{\prime}, \chi_{0}\right) \\
& \otimes \zeta\left(\beta^{\prime}, i^{\prime}+\beta^{\prime}-1, \chi_{0}\right) \rtimes \sigma_{i+\beta-1} .
\end{aligned}\right.
$$

This proves (5.6) as well as the step of the induction.

The next lemma completes the proof that the unramified representation $\sigma$ (Jord) defined by (5.2) is a strongly negative representation. Obviously, by induction on $k$ and $l$ starting from Lemma 5.2, it is enough to prove the next lemma.

Lemma 5.3. Assume that $\operatorname{Jord} \in \operatorname{Jord}(n)$ and $\chi \in\left\{\mathbf{1}, \chi_{0}\right\}$ are given such that $\# \operatorname{Jord}(\chi) \geq 2$. We let $\beta \in \mathbb{Z}_{>0}$ be such that $a=2 \beta+1$ (or $\beta \in \frac{1}{2}+\mathbb{Z}_{\geq 0}$ depending on whether we deal with odd-orthogonal groups or not) is the largest element in $\operatorname{Jord}(\chi)$. We also define $\alpha \in \mathbb{Z}_{\geq 0}$ such that $b=2 \alpha+1$ (or $\alpha \in \frac{1}{2}+\mathbb{Z}_{\geq 0}$ depending on whether we deal with odd-orthogonal groups or not) is the next largest element in $\operatorname{Jord}(\chi)$. Then we put $\operatorname{Jord}^{\prime}=\operatorname{Jord} \backslash\{(a, \chi),(b, \chi)\}$. Put $\sigma^{\prime}=\sigma\left(\operatorname{Jord}^{\prime}\right)$ and $\sigma=\sigma($ Jord $)$. By the construction, we have

$$
\zeta(-\beta, \alpha, \chi) \rtimes \sigma^{\prime} \geq \sigma .
$$

Then if $\sigma^{\prime}$ is strongly negative, $\sigma$ is also strongly negative.

Proof. As in the proof of Lemma 5.1, we show that if $\sigma$ is negative, but not strongly negative, or if $\sigma$ is not negative, leads to a contradiction. First, assume that $\sigma$ is negative, but not strongly negative. Using Theorem 4.4, we can find $2 m \in \mathbb{Z}_{\geq 0}$, an unramified unitary character $\chi^{\prime}$, and an irreducible negative representation $\sigma^{\prime \prime}$ such that

$$
\sigma \hookrightarrow \zeta\left(-m, m, \chi^{\prime}\right) \rtimes \sigma^{\prime \prime} .
$$

Hence, by Frobenius reciprocity, $\mu^{*}(\sigma) \geq \zeta\left(-m, m, \chi^{\prime}\right) \otimes \sigma^{\prime \prime}$. Since $\sigma \leq \zeta(-\beta, \alpha, \chi)$ $\rtimes \sigma^{\prime}$, we see that

$$
\zeta\left(-m, m, \chi^{\prime}\right) \otimes \sigma^{\prime \prime} \leq \mu^{*}\left(\zeta(-\beta, \alpha, \chi) \rtimes \sigma^{\prime}\right) .
$$

Now, using Theorem 3.1, we can find indices $0 \leq j \leq i \leq \alpha+\beta+1$ and an irreducible representation $\mu^{*}\left(\sigma^{\prime}\right) \geq \zeta \otimes \sigma_{1}^{\prime}$ such that

$\zeta\left(-m, m, \chi^{\prime}\right) \otimes \sigma^{\prime \prime} \leq \zeta(-\alpha, \beta-i, \chi) \times \zeta(-\beta, j-\beta-1, \chi) \times \zeta \otimes \zeta(j-\beta, i-\beta-1, \chi) \rtimes \sigma_{1}^{\prime}$.

First, $j=0$ by the construction of $\beta$, since (5.8) implies that any supercuspidal support of $\sigma$ contains $\nu^{\beta} \chi$ or $\nu^{-\beta} \chi$ but not both. Thus,

$$
\zeta\left(-m, m, \chi^{\prime}\right) \otimes \sigma^{\prime \prime} \leq \zeta(-\alpha, \beta-i, \chi) \times \zeta \otimes \zeta(-\beta, i-\beta-1, \chi) \rtimes \sigma_{1}^{\prime} .
$$

If $i=\alpha+\beta+1$, then $\zeta \simeq \zeta\left(-m, m, \chi^{\prime}\right)$. Since $\mu^{*}(\sigma) \geq \zeta\left(-m, m, \chi^{\prime}\right) \otimes \sigma^{\prime \prime}$, Lemma 4.1 contradicts the strong negativity of $\sigma^{\prime}$. Hence $\chi^{\prime}=\chi$ and $m=\alpha$. Next, $\beta-i=\alpha$; otherwise the supercuspidal support of $\sigma^{\prime}$ would contain $\nu^{ \pm \alpha} \chi$, which contradicts the choice of $\sigma^{\prime}$. Now, the above inequality reduces to $\sigma^{\prime \prime} \leq \zeta(-\beta,-\alpha-1, \chi) \rtimes \sigma^{\prime}$. Finally, if we show that $\zeta(-\beta,-\alpha-1, \chi) \rtimes \sigma^{\prime}$ is irreducible, we are done. Using Corollary 4.1, it is enough to show that $\zeta(\alpha+1, \beta, \chi) \rtimes \sigma^{\prime}$ is irreducible. First, we embed $\sigma^{\prime} \hookrightarrow \chi_{1}^{\prime} \times \cdots \times \chi_{l}^{\prime} \rtimes \mathbf{1}$. Note that all $\chi_{i}^{\prime}$ are unramified and by the choice of $\sigma^{\prime},\left|e\left(\chi_{i}^{\prime}\right)\right|<\alpha$. Hence, Lemma 2.1 implies that $\zeta(\alpha+1, \beta, \chi) \times \chi_{i}^{\prime}$ and 
$\zeta(\alpha+1, \beta, \chi) \times \chi_{i}^{\prime}$ are irreducible. Since Theorem 3.2 implies that $\zeta(\alpha+1, \beta, \chi) \rtimes \mathbf{1}$ is irreducible, we see that

$$
\begin{aligned}
\zeta(\alpha+1, \beta, \chi) \rtimes \sigma^{\prime} \hookrightarrow \zeta(\alpha+1, \beta, \chi) \times & \chi_{1}^{\prime} \times \cdots \times \chi_{l}^{\prime} \rtimes \mathbf{1} \\
& \simeq \zeta(-\beta,-\alpha-1, \chi) \times \chi_{1}^{\prime} \times \cdots \times \chi_{l}^{\prime} \rtimes \mathbf{1} .
\end{aligned}
$$

Also, since $\zeta(-\beta,-\alpha-1, \chi) \rtimes \sigma^{\prime} \hookrightarrow \zeta(-\beta,-\alpha-1, \chi) \times \chi_{1}^{\prime} \times \cdots \times \chi_{l}^{\prime} \rtimes \mathbf{1}$, one can complete the proof using the argument from the proof of Lemma 4.8. We leave the details to the reader.

Now, we prove that $\sigma$ is negative. If not, Lemma 4.7 realizes $\sigma$ as follows:

$$
\sigma \hookrightarrow \zeta\left(-\beta_{1}, \alpha_{1}, \chi_{1}\right) \times \cdots \times \zeta\left(-\beta_{k}, \alpha_{k}, \chi_{k}\right) \rtimes \sigma_{n e g},
$$

where the data is as in that lemma. As in the first part of the proof, the Frobenius reciprocity implies

$$
\zeta\left(-\beta_{1}, \alpha_{1}, \chi_{1}\right) \times \cdots \times \zeta\left(-\beta_{k}, \alpha_{k}, \chi_{k}\right) \otimes \sigma_{n e g} \leq \mu^{*}\left(\zeta(-\beta, \alpha, \chi) \rtimes \sigma^{\prime}\right) .
$$

Now, using Theorem 3.1, we can find indices $0 \leq j \leq i \leq \alpha+\beta+1$ and an irreducible representation $\mu^{*}\left(\sigma^{\prime}\right) \geq \zeta \otimes \sigma_{1}^{\prime}$ such that

$$
\begin{aligned}
\zeta\left(-\beta_{1}, \alpha_{1}, \chi_{1}\right) \times \cdots \times \zeta( & \left.-\beta_{k}, \alpha_{k}, \chi_{k}\right) \otimes \sigma_{n e g} \leq \zeta(-\alpha, \beta-i, \chi) \\
\times & \zeta(-\beta, j-\beta-1, \chi) \times \zeta \otimes \zeta(j-\beta, i-\beta-1, \chi) \rtimes \sigma_{1}^{\prime} .
\end{aligned}
$$

First, as in the proof above we show that $i<\alpha+\beta+1$ and $j=0$. Next, Lemma 1.1(ii) also implies that $\zeta$ must be either trivial or unramified. If it is unramified, it is fully induced from the product of the form $\zeta\left(-\beta_{1}^{\prime}, \alpha_{1}^{\prime}, \chi_{1}^{\prime}\right) \times \cdots \times \zeta\left(-\beta_{l}^{\prime}, \alpha_{l}^{\prime}, \chi_{l}^{\prime}\right)$, where all $\chi_{i}^{\prime}$ are unitary and unramified characters, and all $-\beta_{i}^{\prime}+\alpha_{i}^{\prime}<0$ (since $\sigma^{\prime}$ is strongly negative). Finally, by the construction, we see $\beta_{i}^{\prime}<\alpha$. Since $\zeta\left(-\beta_{1}, \alpha_{1}, \chi_{1}\right) \times \cdots \times$ $\zeta\left(-\beta_{k}, \alpha_{k}, \chi_{k}\right)$ is irreducible (see Lemma 4.7) and all $-\beta_{i}+\alpha_{i}>0$, thus, we see $\beta-i \geq \alpha+1, k=1, \zeta$ is trivial. Since $\zeta$ is trivial $\sigma_{1}^{\prime} \simeq \sigma^{\prime}$. Thus the above inequality reduces to $\sigma_{n e g} \leq \zeta(-\beta, i-\beta-1, \chi) \rtimes \sigma^{\prime}$. Exactly as in the first part of the proof, we show that $\zeta(-\beta, i-\beta-1, \chi) \rtimes \sigma^{\prime}$ is irreducible, contradicting the negativity of $\sigma_{\text {neg }}$. This completes the proof of the lemma.

The next lemma will be very important later.

Lemma 5.4. Assume that $\sigma \in \operatorname{Irr} G_{n}$ is a strongly negative unramified representation. Then we have the following:

(i) There exists a unitary unramified character $\chi$ of $F^{\times}, \alpha, \beta \in \mathbb{R}, \alpha+\beta \in \mathbb{Z}_{\geq 0}$, and an irreducible representation $\sigma^{\prime}$, such that

$$
\sigma \hookrightarrow \zeta(-\beta, \alpha, \chi) \rtimes \sigma^{\prime} .
$$

Moreover, assume that $\alpha$ is largest possible (subject to (5.9)); then we have the following:

- $\alpha-\beta<0$.

- $\sigma^{\prime}$ is strongly negative.

- $\zeta(-\beta, \alpha, \chi) \rtimes \sigma^{\prime}$ is reducible.

(ii) Let $\chi$ be an unramified unitary character of $F^{\times}$. Assume that $\beta$ is the largest among all $\left|e\left(\chi^{\prime}\right)\right|$, where $\chi^{\prime}$ appears in the supercuspidal support of $\sigma$ and $\left(\chi^{\prime}\right)^{u}=\chi$. Then $\mu^{*}(\sigma) \geq \nu^{-\beta} \chi \otimes \cdots$. Let $\mu^{*}(\sigma) \geq \zeta(-\beta, \alpha, \chi) \otimes \sigma^{\prime \prime}$, 
where $\alpha, \beta \in \mathbb{R}, \alpha+\beta \in \mathbb{Z}_{\geq 0}$ and $\sigma^{\prime \prime}$ is an irreducible representation such that $\alpha$ is largest possible (with this property). Then there exists an irreducible unramified representation $\sigma^{\prime}$ having the same supercuspidal support as $\sigma^{\prime \prime}$ such that (i) holds.

Proof. We prove (i). First, the existence of embedding of type (5.9) follows from Theorem 2.1. We assume that $\alpha$ is largest possible. Lemma 1.1(ii) forces $\sigma^{\prime}$ to be unramified.

First, $\alpha-\beta<0$ is clear since $\sigma$ is strongly negative. Also, $\zeta(-\beta, \alpha, \chi) \rtimes \sigma^{\prime}$ is reducible. Otherwise using $\widetilde{\sigma} \simeq \sigma$ and $\widetilde{\sigma^{\prime}} \simeq \sigma^{\prime}$, we would get

$$
\sigma \hookrightarrow \zeta\left(-\alpha, \beta, \chi^{-1}\right) \rtimes \sigma^{\prime},
$$

which contradicts strong negativity of $\sigma$. We show that $\sigma^{\prime}$ is strongly negative. This is where we need the assumption that $\alpha$ is the largest possible. If $\sigma^{\prime}$ is not strongly negative, Theorem 4.4 and Lemma 4.7 show that there exists a unitary unramified character $\chi^{\prime}$ of $F^{\times}, \alpha^{\prime}, \beta^{\prime} \in \mathbb{R}, \alpha^{\prime}+\beta^{\prime} \in \mathbb{Z}_{\geq 0}$, and an irreducible unramified representation $\sigma^{\prime \prime}$, such that $\alpha^{\prime}-\beta^{\prime} \geq 0$ and

$$
\sigma^{\prime} \hookrightarrow \zeta\left(-\beta^{\prime}, \alpha^{\prime}, \chi^{\prime}\right) \rtimes \sigma^{\prime \prime} .
$$

We combine (5.9) and (5.10) and look at the following sequence of equivariant morphisms:

$$
\begin{aligned}
\sigma \hookrightarrow \zeta(-\beta, \alpha, \chi) \times \zeta\left(-\beta^{\prime}, \alpha^{\prime}, \chi^{\prime}\right) \rtimes \sigma^{\prime \prime} & \\
& \rightarrow \zeta\left(-\beta^{\prime}, \alpha^{\prime}, \chi^{\prime}\right) \times \zeta(-\beta, \alpha, \chi) \rtimes \sigma^{\prime \prime} .
\end{aligned}
$$

Clearly, $\sigma$ must be in the kernel of the second equivariant morphism. Thus, $\zeta(-\beta, \alpha, \chi) \times \zeta\left(-\beta^{\prime}, \alpha^{\prime}, \chi^{\prime}\right)$ reduces. In particular, $\chi=\chi^{\prime}, \alpha-\alpha^{\prime} \in \mathbb{Z}$. Also one of the following must hold:

- $-\beta<-\beta^{\prime} \leq \alpha+1 \leq \alpha^{\prime}$,

- $-\beta^{\prime}<-\beta \leq \alpha^{\prime}+1 \leq \alpha$.

The second case is not possible since otherwise

$$
0 \leq \alpha^{\prime}-\beta^{\prime} \leq \alpha-\beta<0 .
$$

This discussion implies that the kernel of the second equivariant morphism in (5.11) is isomorphic to

$$
\zeta\left(-\beta, \alpha^{\prime}, \chi\right) \times \zeta\left(-\beta^{\prime}, \alpha, \chi\right) \rtimes \sigma^{\prime \prime},
$$

and as we already observed $\sigma$ embeddes into that kernel. In particular, for some irreducible representation $\sigma_{1}$ we must have

$$
\sigma \hookrightarrow \zeta\left(-\beta, \alpha^{\prime}, \chi\right) \rtimes \sigma_{1} .
$$

Now, Lemma 1.1(ii) implies that $\sigma_{1}$ is unramified, and since $\alpha<\alpha^{\prime}$ this contradicts the maximality of $\alpha$.

Now, we prove (ii). First, by Theorem 2.1, we can find a sequence of representations $\zeta\left(-\beta_{i}, \alpha_{i}, \chi_{i}\right)\left(\alpha_{i}, \beta_{i} \in \mathbb{R}, \alpha_{i}+\beta_{i} \in \mathbb{Z}_{>0}, \chi_{i}\right.$ is an unramified unitary character of $\left.F^{\times}\right), 1 \leq i \leq k$, such that $\zeta\left(-\beta_{1}, \alpha_{1}, \chi_{1}\right) \times \cdots \times \zeta\left(-\beta_{k}, \alpha_{k}, \chi_{k}\right)$ is irreducible and

$$
\sigma \hookrightarrow \zeta\left(-\beta_{1}, \alpha_{1}, \chi_{1}\right) \times \cdots \times \zeta\left(-\beta_{k}, \alpha_{k}, \chi_{k}\right) \rtimes \mathbf{1} .
$$


Note that the assumption that $\sigma$ is strongly negative implies that $\alpha_{i}-\beta_{i}<0$, for all $i$, since $\zeta\left(-\beta_{1}, \alpha_{1}, \chi_{1}\right) \times \cdots \times \zeta\left(-\beta_{k}, \alpha_{k}, \chi_{k}\right)$ is irreducible. Note that $-\beta_{i} \leq$ $\pm \beta \leq \alpha_{i}$, for some $i$, and our assumption on the choice of $\beta$ implies that $\beta_{i}=\beta$. This completes the proof of (ii).

The next lemma tell us more about the position of $\sigma($ Jord $)$. More precisely, we have the following.

Lemma 5.5. Assume that $\sigma$ (Jord) is given by (5.2). Then we have the following:

$$
\begin{aligned}
\sigma(\mathrm{Jord}) \hookrightarrow \zeta\left(-m_{l}, m_{l-1}, \mathbf{1}\right) \times \zeta( & \left.-m_{l-2}, m_{l-3}, \mathbf{1}\right) \times \cdots \times \zeta\left(-n_{k}, n_{k-1}, \chi_{0}\right) \\
& \times \zeta\left(-n_{k-2}, n_{k-3}, \chi_{0}\right) \times \cdots \rtimes \sigma_{0}(\text { Jord }) .
\end{aligned}
$$

In particular, let $\chi \in\left\{\mathbf{1}, \chi_{0}\right\}$ such that $\# \operatorname{Jord}(\chi) \geq 2$. We let $\beta \in \mathbb{Z}_{>0}$ such that $a=2 \beta+1$ (or $\beta \in \frac{1}{2}+\mathbb{Z}_{\geq 0}$ depending on whether we deal with odd-orthogonal groups or not) is the largest element in $\operatorname{Jord}(\chi)$. We also define $\alpha \in \mathbb{Z}_{\geq 0}$ such that $b=2 \alpha+1$ (or $\alpha \in \frac{1}{2}+\mathbb{Z}_{\geq 0}$ depending if we deal with odd-orthogonal groups or not) is the next largest element in $\operatorname{Jord}(\chi)$. Then we put $\operatorname{Jord}^{\prime}=\operatorname{Jord} \backslash\{(a, \chi),(b, \chi)\}$. Put $\sigma^{\prime}=\sigma\left(\right.$ Jord $\left.^{\prime}\right)$ and let $\sigma_{n}^{\prime}$ be the unique unramified irreducible subquotient of $\zeta(-\alpha, \alpha, \chi) \rtimes \sigma^{\prime}$. Then we have the following:

$$
\left\{\begin{array}{l}
\sigma \hookrightarrow \zeta(-\beta, \alpha, \chi) \rtimes \sigma^{\prime}, \\
\sigma_{n}^{\prime} \hookrightarrow \zeta(-\alpha, \alpha, \chi) \rtimes \sigma^{\prime}, \\
\sigma \hookrightarrow \zeta(-\beta,-\alpha-1, \chi) \rtimes \sigma_{n}^{\prime} .
\end{array}\right.
$$

Finally, $\zeta(-\alpha, \alpha, \chi) \rtimes \sigma^{\prime}$ is a direct sum of two non-equivalent self-dual irreducible representations and $\zeta(-\beta, \alpha, \chi) \rtimes \sigma^{\prime}$ has exactly two irreducible subrepresentations. They are self-dual and non-equivalent

Proof. The proof of (5.12) is by induction on $k$ and $l$. The statement is clear if $l=0$ and $k=0$ since $\sigma$ (Jord $) \simeq \sigma_{0}$ (Jord). Assume now that $\chi \in\left\{\mathbf{1}, \chi_{0}\right\}$ such that $\# \operatorname{Jord}(\chi) \geq 2$. We let $\beta \in \mathbb{Z}_{>0}$ such that $a=2 \beta+1$ (or $\beta \in \frac{1}{2}+\mathbb{Z}_{\geq 0}$ depending on whether we deal with odd-orthogonal groups or not) is the largest element in $\operatorname{Jord}(\chi)$. We also define $\alpha \in \mathbb{Z}_{\geq 0}$ to be such that $b=2 \alpha+1$ (or $\alpha \in \frac{1}{2}+\mathbb{Z}_{\geq 0}$ depending on whether we deal with odd-orthogonal groups or not) is the next largest element in $\operatorname{Jord}(\chi)$. Then we put $\operatorname{Jord}^{\prime}=\operatorname{Jord} \backslash\{(a, \chi),(b, \chi)\}$. Put $\sigma^{\prime}=\sigma\left(\right.$ Jord $\left.^{\prime}\right)$. In fact, for definiteness we assume without loss of generality that $\zeta(-\beta, \alpha, \chi)=\zeta\left(-m_{l}, m_{l-1}, \mathbf{1}\right)$. Then the inductive assumption is

$$
\begin{aligned}
\sigma^{\prime} \hookrightarrow \zeta\left(-m_{l-2}, m_{l-3}, \mathbf{1}\right) \times \cdots \times & \zeta\left(-n_{k}, n_{k-1}, \chi_{0}\right) \\
\times & \times\left(-n_{k-2}, n_{k-3}, \chi_{0}\right) \times \cdots \rtimes \sigma_{0}(\text { Jord }) .
\end{aligned}
$$

Now, Theorem 3.2 shows that $\zeta(-\alpha, \alpha, \chi) \rtimes \mathbf{1} \simeq \pi_{1}^{\prime} \oplus \pi_{2}^{\prime}$, where $\pi_{i}^{\prime}, i=1,2$, are irreducible and mutually inequivalent. (We used here the obvious fact that induction carries unitarizable representations into unitarizable representations, Lemma 3.8 (b) of [T1], and the fact that is easy to check that $\zeta(-\alpha, \alpha, \chi) \times \mathbf{1}$ appears 
exactly twice in $\mu^{*}(\zeta(-\alpha, \alpha, \chi) \rtimes \mathbf{1})$.) Combining this with (5.14), we see

$$
\begin{aligned}
\zeta( & -\alpha, \alpha, \chi) \rtimes \sigma^{\prime} \hookrightarrow \zeta(-\alpha, \alpha, \chi) \times \zeta\left(-m_{l-2}, m_{l-3}, \mathbf{1}\right) \\
& \times \cdots \times \zeta\left(-n_{k}, n_{k-1}, \chi_{0}\right) \times \zeta\left(-n_{k-2}, n_{k-3}, \chi_{0}\right) \times \cdots \rtimes \sigma_{0}(\text { Jord }) \\
& \hookrightarrow \zeta(-\alpha, \alpha, \chi) \times \zeta\left(-m_{l-2}, m_{l-3}, \mathbf{1}\right) \times \cdots \times \zeta\left(-n_{k}, n_{k-1}, \chi_{0}\right) \\
& \times \zeta\left(-n_{k-2}, n_{k-3}, \chi_{0}\right) \times \cdots \times \zeta^{\prime} \rtimes \mathbf{1} \\
\quad & \simeq \zeta\left(-m_{l-2}, m_{l-3}, \mathbf{1}\right) \times \cdots \times \zeta\left(-n_{k}, n_{k-1}, \chi_{0}\right) \\
& \times \zeta\left(-n_{k-2}, n_{k-3}, \chi_{0}\right) \times \cdots \times \zeta^{\prime} \times \zeta(-\alpha, \alpha, \chi) \rtimes \mathbf{1} \\
& \simeq \zeta\left(-m_{l-2}, m_{l-3}, \mathbf{1}\right) \times \cdots \times \zeta\left(-n_{k}, n_{k-1}, \chi_{0}\right) \\
& \times \zeta\left(-n_{k-2}, n_{k-3}, \chi_{0}\right) \times \cdots \times \zeta^{\prime} \rtimes\left(\pi_{1}^{\prime} \oplus \pi_{2}^{\prime}\right),
\end{aligned}
$$

where $\widetilde{\zeta^{\prime}}$ is an irreducible representation on the right-hand side of (5.3) depending on type of $\sigma_{0}$ (Jord). (Note that Lemma 5.2 implies $\sigma_{0}($ Jord $) \hookrightarrow \zeta^{\prime} \rtimes \mathbf{1}$.)

Now, it is clear that the following representation is irreducible:

$$
\zeta\left(-m_{l-2}, m_{l-3}, \mathbf{1}\right) \times \cdots \times \zeta\left(-n_{k}, n_{k-1}, \chi_{0}\right) \times \zeta\left(-n_{k-2}, n_{k-3}, \chi_{0}\right) \times \cdots \times \zeta^{\prime} \otimes \pi_{i}^{\prime},
$$

and using Theorem 3.1 it is easy to check that its multiplicity in both

$$
\zeta\left(-m_{l-2}, m_{l-3}, \mathbf{1}\right) \times \cdots \times \zeta\left(-n_{k}, n_{k-1}, \chi_{0}\right) \times \zeta\left(-n_{k-2}, n_{k-3}, \chi_{0}\right) \times \cdots \times \zeta^{\prime} \rtimes \pi_{i}^{\prime}
$$

and

$$
\begin{aligned}
\zeta(-\beta, \beta, \chi) & \times \zeta\left(-m_{l-2}, m_{l-3}, \mathbf{1}\right) \times \cdots \times \zeta\left(-n_{k}, n_{k-1}, \chi_{0}\right) \\
& \times \zeta\left(-n_{k-2}, n_{k-3}, \chi_{0}\right) \times \cdots \times \zeta^{\prime} \rtimes \mathbf{1}
\end{aligned}
$$

is equal to $2^{k+l-1}$, and its multiplicity is at least one in $\zeta(-\alpha, \alpha, \chi) \rtimes \sigma^{\prime}$. We leave simple verification to the reader. Now, (5.15) shows that there exist irreducible representations $\pi_{1}, \pi_{2}$ such that

$$
\pi_{1} \oplus \pi_{2} \hookrightarrow \zeta(-\alpha, \alpha, \chi) \rtimes \sigma^{\prime} .
$$

Now, Theorem 3.1 shows that $\zeta(-\alpha, \alpha, \chi) \otimes \sigma^{\prime}$ appears exactly twice in $\mu^{*}\left(\zeta(-\alpha, \alpha, \chi) \rtimes \sigma^{\prime}\right)$. This shows that either $\pi_{1} \simeq \pi_{2}$ or each $\pi_{i}$ appears with multiplicity one in $\zeta(-\alpha, \alpha, \chi) \rtimes \sigma^{\prime}$, and if $\pi_{3}$ is an irreducible representation such that $\pi_{3} \hookrightarrow(-\alpha, \alpha, \chi) \rtimes \sigma^{\prime}$, then $\pi_{3} \simeq \pi_{1}$ or $\pi_{3} \simeq \pi_{2}$. Next, arguing as in the proof of Lemma 4.8 , we see that $\widetilde{\pi}_{i} \in\left\{\pi_{1}, \pi_{2}\right\}, i=1,2$. Now, taking contragredients and applying Corollary 3.1, we see that (5.16) implies

$$
\zeta(-\alpha, \alpha, \chi) \rtimes \sigma^{\prime} \rightarrow \pi_{1} \oplus \pi_{2} .
$$

The above observation on their multiplicities shows

$$
\zeta(-\alpha, \alpha, \chi) \rtimes \sigma^{\prime} \simeq \pi_{1} \oplus \pi_{2} \oplus W .
$$

Note that $W=0$; otherwise, since it has a finite length we see that it must have an irreducible subrepresentation $\pi_{3} \hookrightarrow W$. This is a contradiction. Thus, $\zeta(-\alpha, \alpha, \chi) \rtimes \sigma^{\prime} \simeq \pi_{1} \oplus \pi_{2}$. Next, we can assume $\sigma_{n} \simeq \pi_{1}$. Then $\pi_{1}$ is self-dual and non-isomorphic to $\pi_{2}$. Thus, $\pi_{2}$ is also self-dual. Thus, $\zeta(-\alpha, \alpha, \chi) \rtimes \sigma^{\prime}$ is a direct sum of two non-equivalent self-dual irreducible representations. Now, we show that $\zeta(-\beta, \alpha, \chi) \rtimes \sigma^{\prime}$ has exactly two irreducible subrepresentations and that 
representations are self-dual and non-equivalent. We have the following chain of embeddings:

$$
\begin{aligned}
\zeta(-\beta, \alpha, \chi) \rtimes \sigma^{\prime} \hookrightarrow \zeta( & -\beta,-\alpha-1, \chi) \times \zeta(-\alpha, \alpha, \chi) \rtimes \sigma^{\prime} \\
& \simeq \zeta(-\beta,-\alpha-1, \chi) \rtimes \pi_{1} \oplus \zeta(-\beta,-\alpha-1, \chi) \rtimes \pi_{2} .
\end{aligned}
$$

Now, using Theorem 3.1, it is not hard to check that $\zeta(-\beta,-\alpha-1, \chi) \otimes \pi_{i}, i=$ 1,2 , appears in $\mu^{*}\left(\zeta(-\beta,-\alpha-1, \chi) \times \zeta(-\alpha, \alpha, \chi) \rtimes \sigma^{\prime}\right)$ and in $\mu^{*}\left(\zeta(-\beta, \alpha, \chi) \rtimes \sigma^{\prime}\right)$ with multiplicity exactly one. We leave the details to the reader. This shows the claim. To complete the proof of the lemma, we use Lemma 5.4(ii) to see that for some irreducible unramified representation $\sigma^{\prime \prime}$ we have

$$
\sigma \hookrightarrow \zeta\left(-\beta, \alpha^{\prime}, \chi\right) \rtimes \sigma^{\prime \prime} .
$$

Taking the maximal possible such $\alpha^{\prime}$ we see that $\sigma^{\prime \prime}$ is strongly negative. Next, if $\alpha=\alpha^{\prime}$ we are done. If not, then $\alpha^{\prime}<\alpha$ because of our choice of $\alpha$ and $\beta$. In any supercuspidal support of $\sigma$ exactly one of the elements $\nu^{ \pm i} \chi,-\alpha \leq i \leq-\beta-1$, appears. We now prove

$$
\sigma \hookrightarrow \zeta(-\beta, \alpha, \chi) \rtimes \sigma^{\prime}
$$

by induction starting from the smallest possible $\beta=\alpha+1$. In that case, $\alpha^{\prime}>-\beta=$ $-\alpha-1$ or otherwise $\sigma^{\prime \prime} \simeq \sigma_{n}$ which is not strongly negative. Now, (5.18) implies

$$
\sigma_{n} \hookrightarrow \zeta(-\alpha, \alpha, \chi) \rtimes \sigma^{\prime} \hookrightarrow \zeta\left(-\alpha, \alpha^{\prime}, \chi\right) \times \zeta\left(\alpha^{\prime}+1, \alpha, \chi\right) \rtimes \sigma^{\prime} .
$$

Now, an obvious analogue of Lemma 4.3 implies

$$
\sigma_{n} \hookrightarrow \zeta\left(-\alpha, \alpha^{\prime}, \chi\right) \rtimes \sigma^{\prime \prime} .
$$

Combining this with (5.18) we get

$$
\sigma \hookrightarrow \zeta\left(-\beta, \alpha^{\prime}, \chi\right) \rtimes \sigma^{\prime \prime} \hookrightarrow \zeta(-\beta,-\alpha-1, \chi) \times \zeta\left(-\alpha, \alpha^{\prime}, \chi\right) \rtimes \sigma^{\prime \prime} .
$$

Hence

$$
\sigma \hookrightarrow \zeta(-\beta,-\alpha-1, \chi) \rtimes \sigma_{n} .
$$

Since $\pi_{1}^{\prime}=\sigma_{n}$, we see $\sigma \simeq \pi_{1}^{\prime}$. This completes the proof of the lemma in this case. In general, for a given $\beta \geq \alpha+1$, we construct $\alpha^{\prime}$. If $\alpha^{\prime} \geq-\alpha$ we argue as before. If $\alpha^{\prime}<-\alpha$, then, as before, $\alpha^{\prime}<-\alpha-1$ and $\sigma^{\prime \prime}$ is strongly negative with $\beta$ replaced by $-\left(\alpha^{\prime}+1\right)$ in Jord for $\sigma$. Now, by the inductive assumption, we see

$$
\sigma^{\prime \prime} \hookrightarrow \zeta\left(\alpha^{\prime}+1, \alpha, \chi\right) \rtimes \sigma^{\prime} .
$$

Combining this with (5.18), we get

$$
\sigma \hookrightarrow \zeta\left(-\beta, \alpha^{\prime}, \chi\right) \rtimes \sigma^{\prime \prime} \hookrightarrow \zeta\left(-\beta, \alpha^{\prime}, \chi\right) \times \zeta\left(\alpha^{\prime}+1, \alpha, \chi\right) \rtimes \sigma^{\prime} .
$$

Now, Lemma 4.3 completes the proof.

The next corollary follows from the proof of Lemma 5.5. We leave an easy proof by induction to the reader.

Corollary 5.1. Assume that $\sigma$ (Jord) is given by (5.12). Assume that we are given a sequence of different characters $\zeta\left(-l_{i}, l_{i}, \chi_{i}\right)$ ( $\chi_{i}$ an unramified unitary character, $2 l_{i} \in \mathbb{Z}_{\geq 0}, \zeta\left(-l_{i}, l_{i}, \chi_{i}\right) \rtimes \mathbf{1}$ reduces $), i=1, \ldots, t$. Moreover assume that $l_{i} \neq m_{j}, n_{k}$ (see (5.2)). Then the induced representation $\zeta\left(-l_{1}, l_{1}, \chi_{1}\right) \times \cdots \times \zeta\left(-l_{t}, l_{t}, \chi_{t}\right) \rtimes$ $\sigma$ (Jord) is isomorphic to the direct sum of $2^{t}$ non-equivalent irreducible representations. 
The next lemma computes some reducibilities. It also proves Theorem 4.2 for the class of strongly negative representations already constructed.

Lemma 5.6. Assume that $\chi_{1}$ is a unitary unramified character of $F^{\times}, \alpha_{1}, \beta_{1} \in \mathbb{R}$, $\alpha_{1}+\beta_{1} \in \mathbb{Z}_{\geq 0}, \alpha_{1}-\beta_{1}>0$. Then we have the following:

(i) $\zeta\left(-\beta_{1}, \alpha_{1}, \chi_{1}\right) \rtimes \mathbf{1}$ reduces if and only if $\nu^{i} \chi_{1} \rtimes \mathbf{1}$ reduces for some $i,-\beta_{1} \leq$ $i \leq \alpha_{1}, \beta_{1}-i \in \mathbb{Z}$. (This is a theorem of Tadić (cf. T1] or Theorem 3.2 here).) More precisely, if $\zeta\left(-\beta_{1}, \alpha_{1}, \chi_{1}\right) \rtimes \mathbf{1}$ reduces, its unique irreducible subrepresentation (see Lemma 4.7) is not unramified, and it has exactly two (resp. one) irreducible quotient(s) if $\beta_{1} \geq 0$ (resp. $\left.\beta_{1}<0\right)$. They are selfdual and non-isomorphic, and one of them is an unramified representation. In fact, they are only irreducible subrepresentations in $\zeta\left(-\alpha_{1}, \beta_{1}, \chi_{1}\right) \rtimes \mathbf{1}$.

(ii) Let $\sigma=\sigma_{\alpha, \alpha^{\prime}}$ be defined as in Lemma 5.2. (We allow $\alpha=\beta-1$ or $\alpha^{\prime}=\beta^{\prime}-1$.) Then $\zeta\left(-\beta_{1}, \alpha_{1}, \chi_{1}\right) \rtimes \sigma$ reduces if and only if one of the following induced representations reduces (see Lemma 2.1):

$$
\left\{\begin{array}{l}
\zeta\left(-\beta_{1}, \alpha_{1}, \chi_{1}\right) \times \zeta(-\alpha,-\beta, \mathbf{1}), \zeta\left(-\alpha_{1}, \beta_{1}, \chi_{1}\right) \times \zeta(-\alpha,-\beta, 1), \\
\zeta\left(-\beta_{1}, \alpha_{1}, \chi_{1}\right) \times \zeta\left(-\alpha^{\prime},-\beta^{\prime}, \chi_{0}\right), \zeta\left(-\alpha_{1}, \beta_{1}, \chi_{1}\right) \times \zeta(-\alpha,-\beta, 1), \\
\zeta\left(-\beta_{1}, \alpha_{1}, \chi_{1}\right) \rtimes \mathbf{1}
\end{array}\right.
$$

unless $\alpha>\beta-1,-\beta_{1}=\beta=1$ and $\chi_{1}=1$. Moreover, if $\zeta\left(-\beta_{1}, \alpha_{1}, \chi_{1}\right) \rtimes \sigma$ reduces, its unique irreducible subrepresentation (see Lemma 4.7 ) is not unramified.

(iii) Assume that $\sigma$ (Jord) is given by (5.2). Then $\zeta\left(-\beta_{1}, \alpha_{1}, \chi_{1}\right) \rtimes \sigma($ Jord $)$ reduces, and its unique irreducible subrepresentation (see Lemma 4.7) is not unramified. This happens if and only if one of the following induced representations reduces:

$$
\left\{\begin{array}{l}
\zeta\left(-\beta_{1}, \alpha_{1}, \chi_{1}\right) \times \zeta\left(-m_{i}, m_{i}, \mathbf{1}\right) \quad \text { for some } i \\
\zeta\left(-\beta_{1}, \alpha_{1}, \chi_{1}\right) \times \zeta\left(-n_{i}, n_{i}, \chi_{0}\right) \quad \text { for some } i, \\
\zeta\left(-\beta_{1}, \alpha_{1}, \chi_{1}\right) \rtimes \sigma_{0}(\text { Jord }) .
\end{array}\right.
$$

Proof. (i) follows from Lemma 5.1 and Lemma 5.5. Now, we prove (ii). Lemma 5.2 implies

$$
\sigma \hookrightarrow \zeta(-\alpha,-\beta, \mathbf{1}) \times \zeta\left(-\alpha^{\prime},-\beta^{\prime}, \chi_{0}\right) \rtimes \mathbf{1} .
$$

Put $\zeta=\zeta(-\alpha,-\beta, \mathbf{1}), \zeta^{\prime}=\zeta\left(-\alpha^{\prime},-\beta^{\prime}, \chi_{0}\right)$, and $\zeta_{1}=\zeta\left(-\beta_{1}, \alpha_{1}, \chi_{1}\right)$. Then we have the following chain of equivariant morphisms:

$$
\begin{aligned}
\zeta_{1} \rtimes \sigma \hookrightarrow \zeta_{1} \times \zeta \times & \zeta^{\prime} \rtimes \mathbf{1} \\
& \rightarrow \zeta \times \zeta_{1} \times \zeta^{\prime} \rtimes \mathbf{1} \rightarrow \zeta \times \zeta^{\prime} \times \zeta_{1} \rtimes \mathbf{1} \\
& \rightarrow \zeta \times \zeta^{\prime} \times \widetilde{\zeta}_{1} \rtimes \mathbf{1} \rightarrow \zeta \times \widetilde{\zeta}_{1} \times \zeta^{\prime} \rtimes \mathbf{1} \rightarrow \widetilde{\zeta_{1}} \times \zeta \times \zeta^{\prime} \rtimes \mathbf{1} .
\end{aligned}
$$

all obtained from the usual equivariant morphisms (cf. [Ze]), except the third one that is obtained from some non-zero map $\zeta_{1} \rtimes \mathbf{1} \rightarrow \widetilde{\zeta}_{1} \rtimes \mathbf{1}$ taking (both) irreducible quotient(s) into (a) subrepresentation(s) (see Lemma 5.5). If all induced representations in (5.20) are irreducible, then all equivariant morphisms in (5.22) are isomorphisms, and we can conclude irreducibility as in the proof of Lemma 4.8. So assume that some induced representations in (5.20) reduce. Assume that the 
first one reduces; then its unique irreducible quotient is unramified and isomorphic to

$$
\zeta\left(-\alpha, \alpha_{1}, \mathbf{1}\right) \times \zeta\left(-\beta_{1},-\beta, \mathbf{1}\right) .
$$

Thus, if the unique irreducible subrepresentation of $\zeta\left(-\beta_{1}, \alpha_{1}, \chi_{1}\right) \rtimes \sigma$ is unramified, then it must be a subquotient of $\zeta\left(-\alpha, \alpha_{1}, \mathbf{1}\right) \times \zeta\left(-\beta_{1},-\beta, \mathbf{1}\right) \times \zeta\left(-\alpha^{\prime},-\beta^{\prime}, \chi_{0}\right) \rtimes \mathbf{1}$, but Lemma 5.5 and Corollary 5.1 show that the subquotient there is negative. This is a contradiction. Similarly we treat the second induced representation in (5.20). Assume now that $\zeta\left(-\beta_{1}, \alpha_{1}, \chi_{1}\right) \rtimes \mathbf{1}$ reduces, but the first two are irreducible. Then the first two equivariant morphisms in (5.22) are isomorphisms. Also, by (i) we have $\chi \in\left\{\mathbf{1}, \chi_{0}\right\}$. Assume first $\beta_{1} \geq 0$. Then our assumption that the first two equivariant morphisms in (5.22) are isomorphisms implies that if the unique irreducible subrepresentation of $\zeta\left(-\beta_{1}, \alpha_{1}, \chi_{1}\right) \rtimes \sigma$ is unramified, then it must be isomorphic to the negative representation (easily obtained from Lemma 5.5) that is a subquotient of $\zeta \times \zeta^{\prime} \times \widetilde{\zeta}_{1} \rtimes \mathbf{1}$. This is a contradiction. Now, assume that $\beta_{1}<0$. For the definiteness we take $\chi=1$. Then using the notation of Lemma 5.2 we must have $\beta_{1}=-\beta$. Note that our assumption that the first induced representation in (5.20) is irreducible forces $\beta_{1}=-1$ (the other possibility $\beta_{1}=-1 / 2$ is removed this way). If $\alpha=\beta-1$ we can argue as before, concluding the reducibility. Thus, we assume $\alpha \neq \beta-1$. We also have

$$
\zeta\left(1, \alpha_{1}, \mathbf{1}\right) \rtimes \sigma \hookrightarrow \zeta\left(1, \alpha_{1}, \mathbf{1}\right) \times \zeta(-\alpha,-1, \mathbf{1}) \times \zeta\left(-\alpha^{\prime},-\beta^{\prime}, \chi_{0}\right) \rtimes \mathbf{1} .
$$

Now, one can use Theorem 3.1 to see that the irreducible representation $\zeta\left(1, \alpha_{1}, \mathbf{1}\right) \times$ $\zeta(-\alpha,-1, \mathbf{1}) \times \zeta\left(-\alpha^{\prime},-\beta^{\prime}, \chi_{0}\right) \otimes \mathbf{1}$ appears in

$$
\mu^{*}\left(\zeta\left(1, \alpha_{1}, \mathbf{1}\right) \times \zeta(-\alpha,-1, \mathbf{1}) \times \zeta\left(-\alpha^{\prime},-\beta^{\prime}, \chi_{0}\right) \rtimes \mathbf{1}\right)
$$

with multiplicity one (resp. two) if $\alpha \neq \alpha_{1}$ (resp. $\alpha=\alpha_{1}$ ). In particular, the induced representation on the right-hand side of (5.23) has a unique irreducible subrepresentation if $\alpha \neq \alpha_{1}$. We consider the case $\alpha=\alpha_{1}$. The other is analogous. Let us write $\pi$ for the unique irreducible subrepresentation of $\zeta(1, \alpha, \mathbf{1}) \rtimes \sigma$. In view of $(5.22)$, it is enough to show that $\pi$ is unramified. Lemma 1.1 shows that this is equivalent to showing that $1 \rtimes \pi$ contains an unramified irreducible subquotient. First, using Lemmas 5.1 and 5.2, we have

$$
\mathbf{1} \rtimes \pi \leq 1 \times \zeta(1, \alpha, \mathbf{1}) \rtimes \sigma \leq \mathbf{1} \times \zeta(1, \alpha, \mathbf{1}) \times \zeta(-\alpha,-1, \mathbf{1}) \rtimes \sigma_{\alpha^{\prime}},
$$

where $\sigma_{\alpha^{\prime}} \hookrightarrow \zeta\left(-\alpha^{\prime},-\beta^{\prime}, \chi_{0}\right) \rtimes \mathbf{1}$. Now, Lemma 5.5 shows that the unramified irreducible subrepresentation $\pi^{\prime} \hookrightarrow \zeta(-\alpha, \alpha, \mathbf{1}) \rtimes \sigma_{\alpha^{\prime}}$ is a unique irreducible unramified subquotient of $\mathbf{1} \times \zeta(1, \alpha, \mathbf{1}) \rtimes \sigma$. Now, the Frobenius reciprocity implies $\mu^{*}\left(\pi^{\prime}\right) \geq \zeta(-\alpha, \alpha, \mathbf{1}) \otimes \sigma_{\alpha^{\prime}}$. Thus, we use Theorem 3.1 to show that $\mu^{*}(\mathbf{1} \rtimes \pi)$ and $\mu^{*}(\mathbf{1} \times \zeta(1, \alpha, \mathbf{1}) \rtimes \sigma)$ contain $\zeta(-\alpha, \alpha, \mathbf{1}) \otimes \sigma_{\alpha^{\prime}}$ with multiplicity two. First, we have

$$
\left\{\begin{array}{l}
\pi \hookrightarrow \zeta(1, \alpha, \mathbf{1}) \rtimes \sigma, \\
\sigma \hookrightarrow \zeta(-\alpha,-1, \mathbf{1}) \rtimes \sigma_{\alpha^{\prime}} .
\end{array}\right.
$$

This implies that

$$
\begin{aligned}
& \mu^{*}(\mathbf{1} \rtimes \pi) \geq(\mathbf{1} \otimes 1+\mathbf{1} \otimes 1) \rtimes \mu^{*}(\pi) \\
& \quad \geq(\mathbf{1} \otimes 1+\mathbf{1} \otimes 1) \rtimes\left(\zeta(1, \alpha, 1) \times \zeta(-\alpha,-1,1) \otimes \sigma_{\alpha^{\prime}}\right) \geq 2 \cdot \zeta(-\alpha, \alpha, 1) \otimes \sigma_{\alpha^{\prime}} .
\end{aligned}
$$

Finally, the other multiplicity can be considered in the same way. 
Going back to (5.22) we see that if one of the induced representations $\zeta(-\alpha,-\beta, \mathbf{1})$ $\times \zeta\left(-\alpha_{1}, \beta_{1}, \chi_{1}\right)$ and $\zeta\left(-\alpha^{\prime},-\beta^{\prime}, \chi_{0}\right) \times \zeta\left(-\alpha_{1}, \beta_{1}, \chi_{1}\right)$ is irreducible, then all other are irreducible in (5.22). Now, one can complete the proof of (ii) as before.

To prove (iii), we observe that using (5.12) and writing an analogue of (5.22), $\zeta\left(-\beta^{\prime}, \alpha^{\prime}, \chi^{\prime}\right) \rtimes \mathbf{1}$ is reducible is necessary condition for reducibility. We leave details to the reader. Now, we use the induction in $k$ and $l$. The basic case $k=l=0$ is (i) and (ii). Thus, to complete the proof of the lemma we show the following. Assume that is given $\operatorname{Jord} \in \operatorname{Jord}(n)$ and $\chi \in\left\{\mathbf{1}, \chi_{0}\right\}$ such that $\# \operatorname{Jord}(\chi) \geq 2$. We let $\beta \in \mathbb{Z}_{>0}$ such that $a=2 \beta+1$ (or $\beta \in \frac{1}{2}+\mathbb{Z}_{\geq 0}$ depending on whether we deal with odd-orthogonal groups or not) is the largest element in $\operatorname{Jord}(\chi)$. We also define $\alpha \in \mathbb{Z}_{>0}$ such that $b=2 \alpha+1$ (or $\alpha \in \frac{1}{2}+\mathbb{Z}_{>0}$ depending on whether we deal with odd-orthogonal groups or not) is the next largest element in $\operatorname{Jord}(\chi)$. Then we put Jord $^{\prime}=$ Jord $\backslash\{(a, \chi),(b, \chi)\}$. Put $\sigma^{\prime}=\sigma\left(\operatorname{Jord}^{\prime}\right)$ and $\sigma=\sigma($ Jord $)$. Lemma 5.5 shows

$$
\sigma \hookrightarrow \zeta(-\beta, \alpha, \chi) \rtimes \sigma^{\prime} .
$$

Now, to complete the proof of Lemma 5.6 we show that $\zeta\left(-\beta^{\prime}, \alpha^{\prime}, \chi^{\prime}\right) \rtimes \sigma$ reduces if and only if its unique irreducible subrepresentation (see Lemma 4.7) is not unramified. This happens if and only if one of the following induced representations reduces:

- $\zeta\left(-\beta^{\prime}, \alpha^{\prime}, \chi^{\prime}\right) \times \zeta(-\beta, \alpha, \chi), \zeta\left(-\alpha^{\prime}, \beta^{\prime},\left(\chi^{\prime}\right)^{-1}\right) \times \zeta(-\beta, \alpha, \chi)$,

- $\zeta\left(-\beta^{\prime}, \alpha^{\prime}, \chi^{\prime}\right) \rtimes \sigma^{\prime}$.

First, we use Lemma 5.5 to write an analogue of (5.22):

$$
\begin{aligned}
& \zeta\left(-\beta^{\prime}, \alpha^{\prime}, \chi^{\prime}\right) \rtimes \sigma \hookrightarrow \zeta\left(-\beta^{\prime}, \alpha^{\prime}, \chi^{\prime}\right) \times \zeta(-\beta, \alpha, \chi) \rtimes \sigma^{\prime} \\
& \rightarrow \zeta(-\beta, \alpha, \chi) \times \zeta\left(-\beta^{\prime}, \alpha^{\prime}, \chi^{\prime}\right) \rtimes \sigma^{\prime} \rightarrow \zeta(-\beta, \alpha, \chi) \times \zeta\left(-\alpha^{\prime}, \beta^{\prime},\left(\chi^{\prime}\right)^{-1}\right) \rtimes \sigma^{\prime} \\
& \rightarrow \zeta\left(-\alpha^{\prime}, \beta^{\prime},\left(\chi^{\prime}\right)^{-1}\right) \times \zeta(-\beta, \alpha, \chi) \rtimes \sigma^{\prime} .
\end{aligned}
$$

As in the first part of the proof we see that the reducibility of $\zeta\left(-\beta^{\prime}, \alpha^{\prime}, \chi^{\prime}\right) \times$ $\zeta(-\beta, \alpha, \chi)$ implies the reducibility of $\zeta\left(-\beta^{\prime}, \alpha^{\prime}, \chi^{\prime}\right) \rtimes \sigma$. Thus we assume that $\zeta\left(-\beta^{\prime}, \alpha^{\prime}, \chi^{\prime}\right) \times \zeta(-\beta, \alpha, \chi)$ is irreducible. Now, if the last one is irreducible, then the reducibility of $\zeta(-\beta, \alpha, \chi) \times \zeta\left(-\alpha^{\prime}, \beta^{\prime},\left(\chi^{\prime}\right)^{-1}\right)$ is treated similarly. Thus, (5.24) shows that the reducibility depends on $\zeta\left(-\beta^{\prime}, \alpha^{\prime}, \chi^{\prime}\right) \rtimes \sigma^{\prime}$. If this induced representation is irreducible one can conclude irreducibility arguing as in Lemma 4.8. Thus, we assume that $\zeta\left(-\beta^{\prime}, \alpha^{\prime}, \chi^{\prime}\right) \times \zeta(-\beta, \alpha, \chi)$ is irreducible and $\zeta\left(-\beta^{\prime}, \alpha^{\prime}, \chi^{\prime}\right) \rtimes \sigma^{\prime}$ is reducible. If $\chi_{1}=\chi$, the first assumption means one of the following holds (see Lemma 2.1):

$$
\left\{\begin{array}{l}
\alpha<\beta \leq \beta^{\prime}<\alpha^{\prime} \\
\beta^{\prime}<\alpha^{\prime} \leq \alpha<\beta
\end{array}\right.
$$

Now, we show that the multiplicity of $\zeta\left(-\beta^{\prime}, \alpha^{\prime}, \chi^{\prime}\right) \times \zeta(-\beta, \alpha, \chi) \otimes \sigma^{\prime}$ in

$$
\mu^{*}\left(\zeta\left(-\beta^{\prime}, \alpha^{\prime}, \chi^{\prime}\right) \times \zeta(-\beta, \alpha, \chi) \rtimes \sigma^{\prime}\right)
$$

is exactly two. Using Theorem 3.1, we take indices $0 \leq j \leq i \leq \alpha+\beta+1$, $0 \leq j^{\prime} \leq i^{\prime} \leq \alpha^{\prime}+\beta^{\prime}+1$ and an irreducible constituent $\mu^{*}\left(\sigma^{\prime}\right) \geq \zeta \otimes \sigma_{1}$ such that $(5.26)$

$$
\left\{\begin{aligned}
\zeta\left(-\beta^{\prime}, \alpha^{\prime}, \chi^{\prime}\right) \times \zeta(-\beta, \alpha, \chi) \leq & \zeta(-\alpha, \beta-i, \chi) \times \zeta(-\beta, j-\beta-1, \chi) \\
& \times \zeta\left(-\alpha^{\prime}, \beta^{\prime}-i^{\prime}, \chi^{\prime}\right) \times \zeta\left(-\beta^{\prime}, j^{\prime}-\beta^{\prime}-1, \chi^{\prime}\right) \times \zeta \\
\sigma^{\prime} \leq \zeta(j-\beta, i-\beta-1, \chi) \times & \zeta\left(j^{\prime}-\beta^{\prime}, i^{\prime}-\beta^{\prime}-1, \chi^{\prime}\right) \rtimes \sigma_{1} .
\end{aligned}\right.
$$


Assume first that the first line in (5.25) holds. Then the first inequality in (5.26) implies that $i^{\prime}=j^{\prime}=\alpha^{\prime}+\beta^{\prime}+1$. Now, the other multiplicities we count exactly as we remarked in the proof of Lemma 5.5. Now, assume that the second line in $(5.25)$ holds. Then the term $\zeta(-\beta, \alpha, \chi)$ can be produced on the right-hand side in the first inequality in (5.26) at least two ways: $i=j=\alpha+\beta+1$ or $i=j=\beta-\alpha$. The desired multiplicity is obtained arguing as in the proof of Lemma 4.8. If $\alpha=\alpha^{\prime}$ there is also a potential third way, $i=\beta-\alpha+1 i=$ $j=\beta-\alpha$. Then $\nu^{\alpha} \chi$ belongs to $\zeta\left(-\alpha^{\prime}, \beta^{\prime}-i^{\prime}, \chi^{\prime}\right) \times \zeta\left(-\beta^{\prime}, j^{\prime}-\beta^{\prime}-1, \chi^{\prime}\right)$. $(\zeta$ itself cannot contribute.) Then it must be produced twice. Hence $\alpha^{\prime}=\beta^{\prime}-i^{\prime}$ and $\alpha^{\prime}=j^{\prime}-\beta^{\prime}-1$. We see $i^{\prime}=-\alpha^{\prime}+\beta^{\prime}<\alpha^{\prime}+\beta^{\prime}+1=j^{\prime}$. This is a contradiction. We write $\pi$ for the unique irreducible subrepresentation of $\zeta\left(-\beta^{\prime}, \alpha^{\prime}, \chi^{\prime}\right) \rtimes \sigma^{\prime}$. It is not unramified by the inductive assumption. As above, we show that the multiplicity of $\zeta\left(-\beta^{\prime}, \alpha^{\prime}, \chi^{\prime}\right) \times \zeta(-\beta, \alpha, \chi) \otimes \sigma^{\prime}$ in $\mu^{*}(\zeta(-\beta, \alpha, \chi) \rtimes \pi)$ is at least two. This implies that the irreducible subrepresentation of $\zeta\left(-\beta^{\prime}, \alpha^{\prime}, \chi^{\prime}\right) \rtimes \sigma$ is a subquotient in $\zeta(-\beta, \alpha, \chi) \rtimes \pi$. Hence it is not unramified. This completes the proof of the lemma.

The next lemma completes our analysis of strongly negative representations. It also proves Theorems 4.2 and 4.3 completely.

Lemma 5.7. Let $n \in \mathbb{Z}_{>0}$. The correspondence $\operatorname{Jord}(n) \rightarrow \operatorname{Irr}_{s n}\left(G_{n}\right)$ given by Jord $\rightsquigarrow \sigma$ (Jord) is surjective.

Proof. Let us denote by $\operatorname{Irr}_{s n}^{\prime}\left(G_{n}\right)$, the set of equivalence classes of strongly negative unramified representations constructed Lemma 5.2 and Lemma 5.5. We prove the lemma by induction. The case $n=1$ follows from Theorem 3.3. Assume that the map $\operatorname{Jord}(m) \rightarrow \operatorname{Irr}_{s n}\left(G_{m}\right)$ given by Jord $\rightsquigarrow \sigma($ Jord $)$ is surjective, and hence bijective, for all $m<n$. Let $\sigma \in \operatorname{Irr}_{s n}\left(G_{n}\right)$. Now, we apply Lemma 5.4(i) and (ii). Thus there exists a unitary unramified character $\chi$ of $F^{\times}, \alpha, \beta \in \mathbb{R}, \alpha+\beta \in \mathbb{Z}_{\geq 0}$, and an irreducible representation $\sigma^{\prime}$, such that

$$
\sigma \hookrightarrow \zeta(-\beta, \alpha, \chi) \rtimes \sigma^{\prime} .
$$

Moreover, we assume that $\alpha$ is largest possible (subject to (5.27)). Then we have the following: $\alpha-\beta<0, \sigma^{\prime} \in \operatorname{Irr}_{s n}\left(G_{n^{\prime}}\right)\left(n^{\prime}<n\right)$ is strongly negative (thus the inductive assumption applies), and $\zeta(-\beta, \alpha, \chi) \rtimes \sigma^{\prime}$ is reducible, that forces $\chi \in\left\{\mathbf{1}, \chi_{0}\right\}$. We also assume that $\beta$ is the largest among all $\left|e\left(\chi^{\prime}\right)\right|$, where $\chi^{\prime}$ appears in the supercuspidal support of $\sigma$ and $\left(\chi^{\prime}\right)^{u}=\chi$. (See Lemma 5.4(ii).)

We want to show that $\alpha$ is strictly greater than any corresponding element "obtained from $\operatorname{Jord}\left(\sigma^{\prime}\right)_{\chi}$ " (see Lemma 5.3 for the meaning of that phrase) if $\operatorname{Jord}\left(\sigma^{\prime}\right)_{\chi} \neq \emptyset$. Since combining Theorem 3.2 and Lemma 5.3, this completes the proof. So, assume $\operatorname{Jord}\left(\sigma^{\prime}\right)_{\chi} \neq \emptyset$. By the inductive assumption, the analogue of Lemma 5.2 or Lemma 5.5 (see (5.12)) for $\sigma^{\prime}$ must hold. Let $\beta_{1}$ be the largest "obtained from Jord $\left(\sigma^{\prime}\right)_{\chi}$ " and $\alpha_{1}$ the next possible or $\alpha_{1}=-\beta$ or $\alpha_{1}=-\beta^{\prime}$ (depending on $\chi$ ) if $\sigma^{\prime}$ is given by Lemma 5.2. We see that for some other strongly negative representation $\sigma^{\prime \prime}$, we must have

$$
\sigma^{\prime} \hookrightarrow \zeta\left(-\beta_{1}, \alpha_{1}, \chi\right) \rtimes \sigma^{\prime \prime}
$$

Now, prove the next claim.

Claim 1. (i) $\alpha-\alpha_{1} \in \mathbb{Z}$.

(ii) Assume $\beta_{1} \geq \alpha$; then $\beta \geq \beta_{1} \geq \alpha \geq \alpha_{1}$. 
(i) follows from Theorem 3.2 and the construction of Jordan blocks from the beginning of the section, applying the inductive assumption for $\sigma^{\prime}$. To prove (ii), we observe that combining (5.27) and (5.28) we obtain:

$$
\sigma \hookrightarrow \zeta(-\beta, \alpha, \chi) \times \zeta\left(-\beta_{1}, \alpha_{1}, \chi\right) \rtimes \sigma^{\prime \prime} .
$$

Now, if $\beta_{1}>\beta$, then the unique irreducible unramified subquotient of $\zeta(-\beta, \alpha, \chi) \times$ $\zeta\left(-\beta_{1}, \alpha_{1}, \chi\right)$ must be fully induced from the product of $\zeta$ 's such that one of them is of the form $\zeta\left(-\beta_{1}, \gamma, \chi\right)$, for some $\gamma$. Then Lemma 4.3 applied to (5.29) implies $\sigma \hookrightarrow \zeta\left(-\beta_{1}, \gamma, \chi\right) \times \cdots$. This contradicts our choice of $\beta$. Now, one can prove $\alpha \geq \alpha_{1}$ similarly by using the maximality of $\alpha$ for fixed $\beta$. This completes the proof of Claim 1 .

Claim 2. Assume $\beta_{1} \geq \alpha, \alpha>\alpha_{1}$ and $\beta>\beta_{1}$.

Assume $\alpha=\alpha_{1}$; then Claim 1 shows $\beta \geq \beta_{1}>\alpha_{1}=\alpha$. Now, we form a new induced representation (see (5.29)):

$$
\sigma \leq \zeta(-\alpha, \alpha, \chi) \times \zeta\left(-\beta, \beta_{1}, \chi\right) \rtimes \sigma^{\prime \prime} .
$$

Now, if $\beta>\beta_{1}$, then, by the inductive assumption and Lemma $5.5, \zeta\left(-\beta, \beta_{1}, \chi\right) \rtimes \sigma^{\prime \prime}$ has an unramified irreducible subrepresentation $\sigma_{1}^{\prime} \in \operatorname{Irr}_{s n}^{\prime}\left(G_{n^{\prime}}\right)$ (this defines $n^{\prime}$ ). Now Corollary 5.1 implies $\sigma \hookrightarrow \zeta(-\alpha, \alpha, \chi) \rtimes \sigma_{1}^{\prime}$. This contradicts the strong negativity of $\sigma$. Similarly, if $\beta=\beta_{1}$, then Corollary 5.1 implies $\sigma \hookrightarrow \zeta(-\alpha, \alpha, \chi) \times$ $\zeta(-\beta, \beta, \chi) \rtimes \sigma^{\prime \prime}$. Again, this contradicts the strong negativity of $\sigma$.

Similarly, we prove $\beta>\beta_{1}$. This completes the proof of the claim.

Claim 3. Assume $\beta_{1} \geq \alpha, \beta_{1}>\alpha$.

This claim has the proof similar to the previous one. Thus, we obtain $\beta>\beta_{1}>$ $\alpha>\alpha_{1}$. Hence by the inductive assumption and Lemma 5.5 we see that

$$
\sigma \hookrightarrow \zeta\left(-\beta, \beta_{1}, \chi\right) \times \zeta\left(-\alpha, \alpha_{1}, \chi\right) \rtimes \sigma^{\prime \prime},
$$

and this contradicts the maximality of $\alpha$. This completes the proof of the lemma.

\section{More on NEGATIVE REPRESEntations}

In this section we want to strengthen Theorem 4.4 .

Theorem 6.1. $\quad$ (i) Assume that $\sigma_{s n}$ is a strongly negative unramified representation and an unramified unitary character $\chi_{G L(n, F)}$. If $\chi^{2} \neq \mathbf{1}$, then $\chi \mathbf{1}_{G L(n, F)} \rtimes \sigma_{s n}$ is irreducible. In particular, $\chi \mathbf{1}_{G L(n, F)} \rtimes \sigma_{s n} \simeq$ $\chi^{-1} \mathbf{1}_{G L(n, F)} \rtimes \sigma_{s n}$.

(ii) Assume that $\sigma \in \operatorname{Irr} G_{n}$ is an unramified representation. Suppose there exist a strongly negative unramified representation $\sigma_{s n}$ and a sequence of unitary characters $\chi_{1} \mathbf{1}_{G L\left(n_{1}, F\right)}, \ldots, \chi_{k} \mathbf{1}_{G L\left(n_{k}, F\right)}$ such that

$$
\sigma \leq \chi_{1} \mathbf{1}_{G L\left(n_{1}, F\right)} \times \cdots \times \chi_{k} \mathbf{1}_{G L\left(n_{k}, F\right)} \rtimes \sigma_{s n} .
$$

Then we have

$$
\sigma \hookrightarrow \chi_{1} \mathbf{1}_{G L\left(n_{1}, F\right)} \times \cdots \times \chi_{k} \mathbf{1}_{G L\left(n_{k}, F\right)} \rtimes \sigma_{s n} .
$$


(iii) Assume that $\sigma \in \operatorname{Irr} G_{n}$ is a negative unramified representation. Then there exist a unique strongly negative unramified representation $\sigma_{s n}$ and a sequence of unitary characters $\chi_{1} \mathbf{1}_{G L\left(n_{1}, F\right)}, \ldots, \chi_{k} \mathbf{1}_{G L\left(n_{k}, F\right)}$ unique up to the permutation and taking inverses such that

$$
\sigma \hookrightarrow \chi_{1} \mathbf{1}_{G L\left(n_{1}, F\right)} \times \cdots \times \chi_{k} \mathbf{1}_{G L\left(n_{k}, F\right)} \rtimes \sigma_{s n} .
$$

Proof. We first prove (i). Note that $\chi \mathbf{1}_{G L(n, F)}=\zeta(-(n-1) / 2,(n-1) / 2, \chi)$. Let us denote by $\sigma$ the unique unramified irreducible subquotient of $\chi \mathbf{1}_{G L(n, F)} \rtimes \sigma_{s n}$. Unfolding $\mu^{*}\left(\chi \mathbf{1}_{G L(n, F)} \rtimes \sigma_{s n}\right)$ using Theorem 3.1, we see that $\sigma$ must be negative. The classification of strongly negative representations from the previous section show that it must be negative but not strongly negative. (We compare supercuspidal supports of $\sigma_{s n}$ and $\sigma$ and use Lemma 5.3.) Again, using Theorem 3.1 , since $\mu^{*}(\sigma) \leq \mu^{*}\left(\chi \mathbf{1}_{G L(n, F)} \rtimes \sigma_{s n}\right)$, we see that $\mu^{*}(\sigma) \geq \chi \mathbf{1}_{G L(n, F)} \otimes \sigma_{s n}$ or $\mu^{*}(\sigma) \geq \chi^{-1} \mathbf{1}_{G L(n, F)} \otimes \sigma_{s n}$ (contained in $\mu^{*}\left(\chi \mathbf{1}_{G L(n, F)} \rtimes \sigma_{s n}\right)$ with multiplicity one). Thus, by the Frobenius reciprocity, $\sigma \hookrightarrow \chi \mathbf{1}_{G L(n, F)} \rtimes \sigma_{s n}$ or $\sigma \hookrightarrow$ $\chi^{-1} G L(n, F) \rtimes \sigma_{s n}$ as the unique irreducible subrepresentation. If we show that both hold, then $\chi \mathbf{1}_{G L(n, F)} \rtimes \sigma_{s n}$ is irreducible. In more detail, if $\pi$ is an irreducible quotient of $\chi \mathbf{1}_{G L(n, F)} \rtimes \sigma_{s n}$, then $\widetilde{\pi} \hookrightarrow \chi^{-1} G L(n, F) \rtimes \sigma_{s n}$. Thus, $\widetilde{\pi} \simeq \sigma$. Hence Corollary 3.1 implies $\pi \simeq \sigma$. Since $\chi \mathbf{1}_{G L(n, F)} \rtimes \sigma_{s n}$ has the unique irreducible subrepresentation and it contains $\sigma$ with multiplicity one, $\chi \mathbf{1}_{G L(n, F)} \rtimes \sigma_{s n}$ must be irreducible. Finally, we have the following chain of equivariant morphisms:

$$
\begin{aligned}
\chi \mathbf{1}_{G L(n, F)} \rtimes \sigma_{s n} \hookrightarrow \nu^{-(n-1) / 2} \chi \times \cdots \times \nu^{(n-1) / 2} \chi \rtimes \sigma_{s n} \\
\simeq \nu^{-(n-1) / 2} \chi \times \cdots \times \nu^{(n-1) / 2-1} \chi \times \nu^{-(n-1) / 2} \chi^{-1} \rtimes \sigma_{s n} \\
\simeq \nu^{-(n-1) / 2} \chi \times \cdots \times \nu^{-(n-1) / 2} \chi^{-1} \times \nu^{(n-1) / 2-1} \chi \rtimes \sigma_{s n} \\
\cdots \\
\simeq \nu^{-(n-1) / 2} \chi^{-1} \times \nu^{-(n-1) / 2} \chi \times \cdots \times \nu^{(n-1) / 2-1} \chi \rtimes \sigma_{s n} \\
\cdots \\
\simeq \nu^{-(n-1) / 2} \chi^{-1} \times \cdots \times \nu^{(n-1) / 2} \chi^{-1} \rtimes \sigma_{s n} .
\end{aligned}
$$

Also, since

$$
\chi \mathbf{1}_{G L(n, F)} \rtimes \sigma_{s n} \hookrightarrow \nu^{-(n-1) / 2} \chi^{-1} \times \cdots \times \nu^{(n-1) / 2} \chi^{-1} \rtimes \sigma_{s n},
$$

we see $\sigma \hookrightarrow \chi \mathbf{1}_{G L(n, F)} \rtimes \sigma_{s n}$ and $\sigma \hookrightarrow \chi^{-1} G L(n, F) \rtimes \sigma_{s n}$ as unique irreducible subrepresentations.

Now, we prove (ii). Let $\sigma \in \operatorname{Irr} G_{n}$. Again, using Theorem 3.1 and the classification of strongly negative representations, it follows that $\sigma$ is negative and, more precisely, we have

$$
\begin{aligned}
\operatorname{Jacq}_{\left(n_{1}+\cdots+n_{k} ; n-n_{1}-\cdots-n_{k}\right)}(\sigma) & \\
& \simeq \operatorname{Jacq}_{\left(n_{1}+\cdots+n_{k} ; n-n_{1}-\cdots-n_{k}\right)}(\sigma)^{u} \oplus \operatorname{Jacq}_{\left(n_{1}+\cdots+n_{k} ; n-n_{1}-\cdots-n_{k}\right)}(\sigma)^{n u},
\end{aligned}
$$

into parts that have unitary and non-unitary generalized central characters (with respect to the center of $G\left(n_{1}+\cdots+n_{k}, F\right)$. Using Theorem 3.1 it is easy to see that all irreducible subquotients of $\operatorname{Jacq}_{\left(n_{1}+\cdots+n_{k} ; n-n_{1}-\cdots-n_{k}\right)}(\sigma)^{u}$ are of the form

$$
\mu^{*}(\sigma) \geq \chi_{1}^{\epsilon_{1}} \mathbf{1}_{G L\left(n_{1}, F\right)} \times \cdots \times \chi_{k}^{\epsilon_{k}} \mathbf{1}_{G L\left(n_{k}, F\right)} \otimes \sigma_{s n},
$$


for some $\epsilon_{i} \in\{ \pm 1\}$. In particular, one of them must satisfy

$$
\operatorname{Jacq}_{\left(n_{1}+\cdots+n_{k} ; n-n_{1}-\cdots-n_{k}\right)}(\sigma) \rightarrow \chi_{1}^{\epsilon_{1}} \mathbf{1}_{G L\left(n_{1}, F\right)} \times \cdots \times \chi_{k}^{\epsilon_{k}} \mathbf{1}_{G L\left(n_{k}, F\right)} \otimes \sigma_{s n} .
$$

Hence the Frobenius reciprocity implies

$$
\sigma \hookrightarrow \chi_{1}^{\epsilon_{1}} \mathbf{1}_{G L\left(n_{1}, F\right)} \times \cdots \times \chi_{k}^{\epsilon_{k}} \mathbf{1}_{G L\left(n_{k}, F\right)} \rtimes \sigma_{s n} .
$$

Now, using isomorphism $\chi \mathbf{1}_{G L(n, F)} \times \chi^{\prime} \mathbf{1}_{G L\left(n^{\prime}, F\right)} \simeq \chi^{\prime} \mathbf{1}_{G L\left(n^{\prime}, F\right)} \times \chi \mathbf{1}_{G L(n, F)}\left(\chi, \chi^{\prime}\right.$ are unitary unramified characters) and (i), (6.1) can be transformed into

$$
\sigma \hookrightarrow \chi_{1} \mathbf{1}_{G L\left(n_{1}, F\right)} \times \cdots \times \chi_{k} \mathbf{1}_{G L\left(n_{k}, F\right)} \rtimes \sigma_{s n} .
$$

Finally, (ii) follows (i) arguing as in the proof of Lemma 4.7. We leave the details to the reader.

\section{REFERENCES}

[B] D. Ban, Parabolic induction and Jacquet modules of representations of $O(2 n, F)$, Glas. Mat. Ser III 34(54)(2) (1999), 147-185. MR1739616 (2001m:22033)

[BM1] D. Barbasch, A. Moy, Whittaker models with an Iwahori fixed vector, Representation theory and analysis on homogenuos spaces (New Brunswick, NJ, 1993) Amer. Math. Soc., Providence, Rhode Island (1994), 101-105. MR.1303602 (95j:22024)

[BM2] D. Barbasch, A. Moy, A unitarity criterion for p-adic groups, Invent. Math. 98 (1989), 19-37. MR.1010153 (90m:22038)

[BM3] D. Barbasch, A. Moy, Reduction to real infinitensimal character in affine Hecke algebras, Journal of A.M.S. 6 (1993), 611-635. MR.1186959 (93k:22015)

[BZ1] I. N. Bernstein, A. V. Zelevinsky, Induced representations of reductive p-adic groups I, Ann. Sci. École Norm Sup. 10 (1977), 441-472. MR0579172 (58:28310)

[BZ2] I. N. Bernstein, A. V. Zelevinsky, Representations of the group $G L(n, F)$, where $F$ is a local non-archimedean field, Uspekhi Mat. Nauk 31 (1976), 5-70. MR0425030(54:12988)

[Car] P. Cartier, Representations of p-adic groups: a survey. Automorphic forms, representations and L-functions (Proc. Sympos. Pure Math., Oregon State Univ., Corvallis, Ore., 1977), Part 1, vol. XXXIII, Proc. Sympos. Pure Math., Amer. Math. Soc., Providence, R.I, 1979, pp. 111-155. MR0546593 (81e:22029)

[KL] D. Kazhdan, G. Lusztig, Proof of the Deligne-Langlands conjecture for Hecke algebras, Invent. Math. 87 (1987), 153-215. MR0862716 (88d:11121)

$[\mathrm{Ku}]$ S. S. Kudla, On the Theta Correspondence (lectures at European School of Group Theory, Beilngries, 1996).

[M1] G. Muić, Some results on square integrable representations; Irreducibility of standard representations, Internat. Math. Res. Notices 14 (1998), 705-726. MR1637097(99f:22031)

[M2] G. Muić, A proof of Casselman-Shahidi's conjecture for quasi-split classical groups, Canad. Math. Bull. 44 (2001), 298-312. MR.1847492 (2002f:22015)

[M3] G. Muić, On generic irreducible representations for $S p(n, F)$ and $S O(2 n+1, F)$, Glas. Mat. Ser III 33(53) (1988), 19-31. MR.1652772 (2000j:22020)

[MSh] G. Muić, F. Shahidi, Irreducibility of standard representations for Iwahori-spherical representations, Math. Ann. 312 (1998), 151-165. MR.1645956 (99g:22012)

[Moe] C. Moeglin, Sur la classification des séries discrètes des groupes classiques p-adiques: paramètres de Langlands et exhaustivité, J. Eur. Math. Soc. (JEMS) J. Eur. Math. Soc. (JEMS) 4 (2002), 143-200. MR1913095 (2003g:22021)

[MT] C. Moeglin, M. Tadić, Construction of discrete series for classical p-adic groups, Amer. J. Math. Soc. 15 (2002), 715-786. MR.1896238 (2003g:22020)

[MVW] C. Moeglin, M.-F. Vignéras, J.-L. Waldspurger, Correspondence de Howe sur un corps p-adique, Lecture Notes in Math. 1291, 1987. MR1041060 (91f:11040)

[Ra] S. Rallis, Langland's functoriality and the Weil representation, Amer. J. Math. 104 (1982), 469-515. MR0658543 (84c:10025)

[Sh1] F. Shahidi, A proof of Langland's conjecture on Plancherel measures; complementary series for p-adic groups, Ann. of Math. 132 (1990), 273-330. MR:1070599 (91m:11095)

[Sh2] F. Shahidi, Twisted endoscopy and reducibility of induced representations for p-adic groups, Duke Math. J. 66 (1992), 1-41. MR.1159430 (93b:22034) 
[T1] M. Tadić, On reducibility of parabolic induction, Israel J. Math. 107 (1998), 29-91. MR 1658535 (2001d:22012)

[T2] M. Tadić, Structure arising from induction and Jacquet modules of representations of classical p-adic groups, Journal of Algebra 177 (1995), 1-33. MR1356358 (97b:22023)

[T3] M. Tadić, A familiy of square-integrable representations of classical p-adic groups in the case of general half-integral reducibilities groups, Glas. Mat. Ser. III 37 (2002), 21-57. MR $1918092(2004 \mathrm{a}: 22017)$

[Ze] A. V. Zelevinsky, Induced representations of reductive p-adic groups. On irreducible representations of $G L(n)$, Ann. Sci. Ecole Norm. Sup. 13 (1980), 165-210. MR0584084 (83g:22012)

Department of Mathematics, University of Zagreb, Bijenicka 30, 10000 Zagreb, CroaTIA

E-mail address: gmuic@math.hr 